RESEARCH ARTICLE SUMMARY

\title{
NEURODEVELOPMENT
}

\section{Spatiotemporal structure of cell fate decisions in murine neural crest}

\author{
Ruslan Soldatov*, Marketa Kaucka*, Maria Eleni Kastriti*, Julian Petersen, \\ Tatiana Chontorotzea, Lukas Englmaier, Natalia Akkuratova, Yunshi Yang, \\ Martin Häring, Viacheslav Dyachuk, Christoph Bock, Matthias Farlik, \\ Michael L. Piacentino, Franck Boismoreau, Markus M. Hilscher, Chika Yokota, \\ Xiaoyan Qian, Mats Nilsson, Marianne E. Bronner, Laura Croci, Wen-Yu Hsiao, \\ David A. Guertin, Jean-Francois Brunet, Gian Giacomo Consalez, Patrik Ernfors, \\ Kaj Fried, Peter V. Kharchenko $\dagger$, Igor Adameyko $\dagger$
}

INTRODUCTION: Multipotent progenitors must choose among multiple downstream fates. In developing embryos, progenitor cells exhibit transcriptional or epigenetic heterogeneity that is related to early biases in cell fate choices, and can be externally induced or stochastic in nature. Molecular assessment of the transient states assumed by cells during these developmental progressions has the potential to illuminate how such fatespecific biases emerge and unfold to ensure fate commitment. With this aim, we examine multipotent neural crest cells-transient embryonic progenitors unique to vertebrates that build the head, teeth, neuroendocrine tissue, and autonomic and sensory nervous systems. Cranial neural crest preferentially

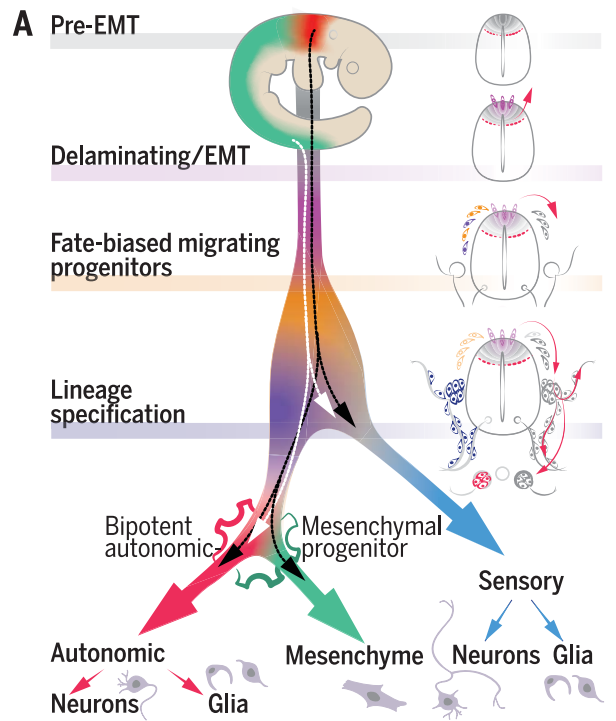

gives rise to a multitude of mesenchymal types of facial cartilage and bones, in addition to neuronal, glial, and pigment cell-type progeny. By contrast, trunk neural crest does not form bone or cartilage derivatives in vivo. The logic and molecular mechanisms that allow neural crest to resolve multiple potential cell fates at each axial level remain poorly understood.

RATIONALE: Here we used single-cell and spatial transcriptomics with statistical analysis of branching trajectories to investigate lineage relationships in mouse neural crest. Combined with lineage tracing and functional perturbations, we addressed spatiotemporal dynamics associated with early cell fate decisions in

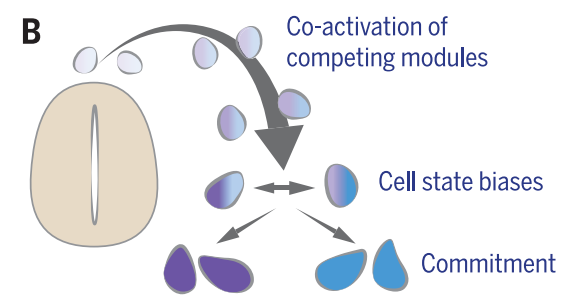

C

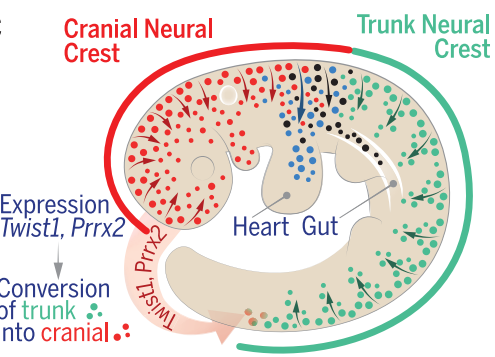

Cell fate acquisition in neural crest. (A) Stepwise model of neural crest developmental dynamics. EMT, epithelial-to-mesenchymal transition. (B) Key phases of fate decision in neural crest cells. (C) Different fate potential of cranial versus trunk neural crest is encoded by the mesenchymogenic genes Twist1 and Prrx2. A single factor, Twist1, confers mesenchymal potential to trunk neural crest.

mouse trunk and cranial neural crest cells with different fate potential.

RESULTS: We find that up to early migration, neural crest cells progress through a sequence of common transcriptional states, followed by fate bifurcations during migration that can be formalized as a series of sequential binary decisions. The first decision separates sensory neuro-glial fate from all other fates, whereas the second decision occurs between autonomic and mesenchymal lineages and reveals a bipotent $P$ hox $2 b^{+} /{\text {Prr } x 1^{+}}^{+}$subpopulation. De-

ON OUR WEBSITE

Read the full article at http://dx.doi. org/10.1126/ science.aas 9536 \section{later steps. Each decision} consists of initial coactivation, gradual biasing, and commitment phases. Early genes of competing cell fate programs coactivate in the same cells, starting from premigratory stage. As cells approach cell fate bifurcation points, increased synchronization of fate-specific programs and repulsion of competing fate programs lead to gradual appearance of cell fate bias, which becomes pronounced upon neural crest migration. Cell fate commitment culminates with activation of mutually exclusive, fate-specific gene expression programs. Early transcriptional patterns reveal that fate biasing of neural crest is already detectable when neural crest cells delaminate from the neural tube. In particular, the neuronal bias of trunk and mesenchymal bias of cranial neural crest emerge during delamination, indicating that this might be the time when the mesenchymal potential, distinct between cranial and trunk neural crest, is installed. In support to this hypothesis, we find that sustained overexpression of a single gene, Twist1, normally activated upon delamination only in the cranial compartment, is sufficient to reverse the trunk crest developmental program to a mesenchymal route.

CONCLUSION: Our analysis resolved a branching transcriptional trajectory of the differentiating neural crest, illustrating transcriptional implementation of major cell fate decisions and pinpointing the key differences defining cranial versus trunk neural crest potential. Our results show that neural crest cells differentiate through a series of stereotypical lineage-restriction events that involve coexpression and competition of genes driving alternative fate programs.

The list of author affiliations is available in the full article online. *These authors contributed equally to this work.

†Corresponding author. Email: igor.adameyko@ki.se (I.A.); peter.kharchenko@post.harvard.edu (P.V.K.)

Cite this article as R. Soldatov et al., Science 364 eaas9536 (2019). DOI: 10.1126/science.aas9536 
RESEARCH ARTICLE

NEURODEVELOPMENT

\section{Spatiotemporal structure of cell fate decisions in murine neural crest}

\author{
Ruslan Soldatov $^{1 *}$, Marketa Kaucka $^{2,3 *}$, Maria Eleni Kastriti $^{2,3 *}$, Julian Petersen $^{2,3}$, \\ Tatiana Chontorotzea ${ }^{3}$, Lukas Englmaier ${ }^{2}$, Natalia Akkuratova ${ }^{3,4}$, Yunshi Yang ${ }^{3}$, \\ Martin Häring 5 , Viacheslav Dyachuk ${ }^{6,7}$, Christoph Bock ${ }^{8,9,10}$, Matthias Farlik ${ }^{8}$, \\ Michael L. Piacentino ${ }^{11}$, Franck Boismoreau ${ }^{12}$, Markus M. Hilscher ${ }^{5,13}$, Chika Yokota ${ }^{13}$, \\ Xiaoyan Qian ${ }^{13,14}$, Mats Nilsson ${ }^{13}$, Marianne E. Bronner ${ }^{11}$, Laura Croci ${ }^{15}$, \\ Wen-Yu Hsiao ${ }^{16}$, David A. Guertin ${ }^{16}$, Jean-Francois Brunet ${ }^{12}$, Gian Giacomo Consalez ${ }^{15}$, \\ Patrik Ernfors $^{5}$, Kaj Fried ${ }^{6}$, Peter V. Kharchenko ${ }^{1,17}+$, Igor Adameyko ${ }^{2,3} \dagger$
}

Neural crest cells are embryonic progenitors that generate numerous cell types in vertebrates. With single-cell analysis, we show that mouse trunk neural crest cells become biased toward neuronal lineages when they delaminate from the neural tube, whereas cranial neural crest cells acquire ectomesenchyme potential dependent on activation of the transcription factor Twist1. The choices that neural crest cells make to become sensory, glial, autonomic, or mesenchymal cells can be formalized as a series of sequential binary decisions. Each branch of the decision tree involves initial coactivation of bipotential properties followed by gradual shifts toward commitment. Competing fate programs are coactivated before cells acquire fate-specific phenotypic traits. Determination of a specific fate is achieved by increased synchronization of relevant programs and concurrent repression of competing fate programs.

M ultipotent progenitors acquire one of the multiple downstream fates. In developmental systems, progenitor cells exhibit transcriptional or epigenetic heterogeneity related to early biases in cell fate choices and can be oscillatory or stochastic (1-3). Understanding how fate-specific biases emerge and unfold to ensure fate commitment requires

Department of Biomedical Informatics, Harvard Medical School, Boston, MA 02115, USA. ²Department of Molecular Neurosciences, Center for Brain Research, Medical University Vienna, 1090 Vienna, Austria. ${ }^{3}$ Department of Physiology and Pharmacology, Karolinska Institutet, 17177 Stockholm, Sweden. ${ }^{4}$ Institute of Translational Biomedicine, St Petersburg University, 199034 St Petersburg, Russia. ${ }^{5}$ Department of Medical Biochemistry and Biophysics, Division of Molecular Neurobiology, Karolinska Institutet, 17177 Stockholm, Sweden. ${ }^{6}$ Department of Neuroscience, Karolinska Institutet, 17177 Stockholm, Sweden. ${ }^{7}$ National Scientific Center of Marine Biology, Far Eastern Branch, Russian Academy of Sciences, Vladivostok 690041, Russia. ${ }^{8} \mathrm{CeMM}$ Research Center for Molecular Medicine of the Austrian Academy of Sciences, Vienna, Austria. ${ }^{9}$ Department of Laboratory Medicine, Medical University of Vienna, Vienna, Austria. ${ }^{10}$ Max Planck Institute for Informatics, Saarbrücken, Germany. ${ }^{11}$ Division of Biology and Biological Engineering California Institute of Technology, Pasadena, CA 91125, USA ${ }^{12}$ Institut de Biologie de I'ENS (IBENS), INSERM, CNRS, École Normale Supérieure, PSL Research University, 75005 Paris, France. ${ }^{13}$ Science for Life Laboratory, Department of Biophysics and biochemistry, Stockholm University, 17165 Solna, Sweden. ${ }^{14}$ Cartana AB, 17165 Solna, Sweden. ${ }^{15}$ San Raffaele Scientific Institute and Università Vita-Salute San Raffaele, 20132, Milan, Italy. ${ }^{16}$ Program in Molecular Medicine, University of Massachusetts Medical School, Worcester, MA 01605, USA ${ }^{17}$ Harvard Stem Cell Institute, Cambridge, MA 02138, USA. *These authors contributed equally to this work.

†Corresponding author. Email: igor.adameyko@ki.se (I.A.); peter.kharchenko@post.harvard.edu (P.V.K.) deeper understanding of cell profiles during progenitor, transient, and derivative states. Toward that goal, we examined a continuum of states in the neural crest (NC) lineage at different points along the murine rostrocaudal body axis during embryogenesis. As neural crest cell fate decisions are traceable, irreversible, and produce wellknown differentiated cell types, we were able to investigate the interplay of multiple fate-specific genetic programs.

Multipotent neural crest cells are a migratory embryonic cell population found only in vertebrates that confers traits such as complex head, teeth, elaborate endocrine regulation, and autonomic and sensory nervous systems. Neural crest cells have distinct developmental potential along the anterior-posterior axis. Cranial neural crest cells give rise to mesenchymal cell types of the head, including facial cartilage and bone, glia, ment cells (4). By contrast, trunk neural crest cells do not form bone or cartilage derivatives in vivo, even after being grafted to the head (5). However, despite knowledge about genes and signals that regulate neural crest development (6), the mechanisms that enable neural crest cells to commit to a multitude of possible cell fates at each axial level remain unclear.

Here we combine single-cell RNA sequencing (scRNA-seq) with spatial transcriptomics and lineage tracing to examine the spatiotemporal involved in cranial and trunk neural crest differentiation in the mouse embryo at the embryonic day 8.5 (E8.5) to E10.5 stages. and some neurons of cranial ganglia and pigtranscriptional landscape and cell fate decisions
ScRNA-seq reveals the transcriptional changes in neural crest during delamination

$W n t 1^{\text {Cre }}$ recombines in the dorsal neural tube where the premigratory neural crest resides. Focusing first on trunk neural crest, we dissected, then dissociated, the cervical region and trunk areas posterior to the otic vesicle from E9.5 $W_{n t 1}{ }^{\text {Cre }} /$ R2 $6 R^{\text {Tomato }}$ mouse embryos (Fig. 1A), and measured mRNAs of single TOMATO $^{+}$cells with high coverage using Smart-seq2 protocol (median of 7025 genes detected per cell) (fig. S1A). Our design takes advantage of the developmental asynchrony of neural crest formation along the anteroposterior axis of the embryo to sample cellular states along neural crest maturation trajectories.

Analysis of transcriptional heterogeneity (7) separates neural tube and neural crest populations into two compartments (6) (Fig. 1, B, C, and E, and fig. S1, F and G). These are connected by two "bridges," corresponding to neural crest delamination [marked by activation of epithelialto-mesenchymal transition (EMT) drivers such as Snai1] (6) (Fig. 1, G to I, and fig. S1C) and neurogenesis processes [marked by proneurogenic transcription factors (TFs)] (fig. S2, B to E). Estimates of RNA velocity, which predict the direction in which cells are moving in transcriptional space (8), show a convergent velocity pattern in the neurogenesis bridge, reflecting convergence of neural crest and neural tube neurogenesis programs (Fig. 1D). By contrast, the delaminating bridge shows pronounced movement from the neural tube toward differentiating neural crest cells. Although delamination of neural crest has been viewed as an abrupt transition of pre-EMT to migrating neural crest (9), our data reveal an extensive sequence of transcriptional events that unfold during delamination and early migration (Fig. 1, B, H, and I; fig. S1C; and table S2).

This enabled us to separate the premigratory neural crest into two distinct subpopulations. The earliest, pre-EMT population, is composed of cells that have not yet started delaminating from the neural tube. It is marked by peak expression of neural plate border specifiers previously tied to NC, such as Zic1/3/5, MsxI $(9,10)$, $M a f b$ (11), and Gdf7 (12) (Fig. 1, E and G, and fig. $\mathrm{S1C})$. The pre-EMT population, however, still expresses neural tube markers such as Olig3 and FoxB1 and is localized to the neural tube (Fig. $1 \mathrm{H}$ and fig. S1, C and F). The second, delaminating subpopulation is marked by activation of the key EMT gene Snai1 (6) and absence of Atoh1, and is accompanied by sequential transient upregulation of a battery of genes, including $D l x 5$, Pak3, Pdgfra, and Hapln (Fig. 1H,I). Up-regulation of classical neural crest specifiers (Sox9, Foxd3, and Ets1) and down-regulation of many neural plate border specifiers (Zic3, Mafb, Gdf7) show variable timing across this progression, with some neuroectodermal border specifiers, such as MsxI, Msx 2, Wnt3a, and Lmx1a (9,13, 14), retaining expression in the delamination cluster. On the side opposite the neural tube, the delamination bridge connects with a subpopulation of $S o x 9^{+}$ 
Fig. 1. Major aspects of trunk neural crest heterogeneity. (A) Immunohistochemistry of E9.5 Wnt1 ${ }^{\mathrm{Cre}}$,R26R ${ }^{\mathrm{TOM}+}$ embryo, showing SOX $10^{+} /$Wnt1 $^{\text {TOM+ }}$ neural crest cells (NCCs) migrating in the head and trunk. Note post-otic vesicle incision (dashed lines), showing separation between cranial and trunk portions. (B and $\mathbf{C}$ ) t-SNE embedding shows 1107 cells (points) from mouse E9.5 Wnt1 ${ }^{\text {Cre }} / R_{2} 26 R^{\text {Tomato }}$

trunk. The embedding reflects spatiotemporal aspects of neural crest (NC) development. Twelve major clusters of transcriptionally similar cells (colors) correspond to different stages of $\mathrm{NC}$ and ventral neural tube (vNT) development. (D) Analysis of RNA velocity shows major directions of cell progression in transcriptional space. The arrow start and end points indicate observed-current and predicted-future cell states, respectively. (E) Gene markers (clusterspecific) link NC clusters to developmental states.

(F) Anatomical localization of NC clusters inferred from in situ sequencing of 32 cluster-specific genes in E9.5 sections (representative trunk section). Cell identity of VNT, which was not captured in the single-cell dataset, is imputed using the observed NC and NT clusters. (G) Characteristic gene expression patterns as detected by scRNA-seq (upper) and in situ sequencing (bottom) of a representative trunk section (see data S11 for others). (H) Transcriptional dynamics around delamination illustrate relationships between previously unknown and known markers. Cells of pre-EMT, delaminatory, and early migratory clusters were ordered by their delamination pseudotime, estimated using a principal curve. (I) scRNA-seq data predicts specific DIx5 expression around delamination (left), validated through immunochemistry (right).
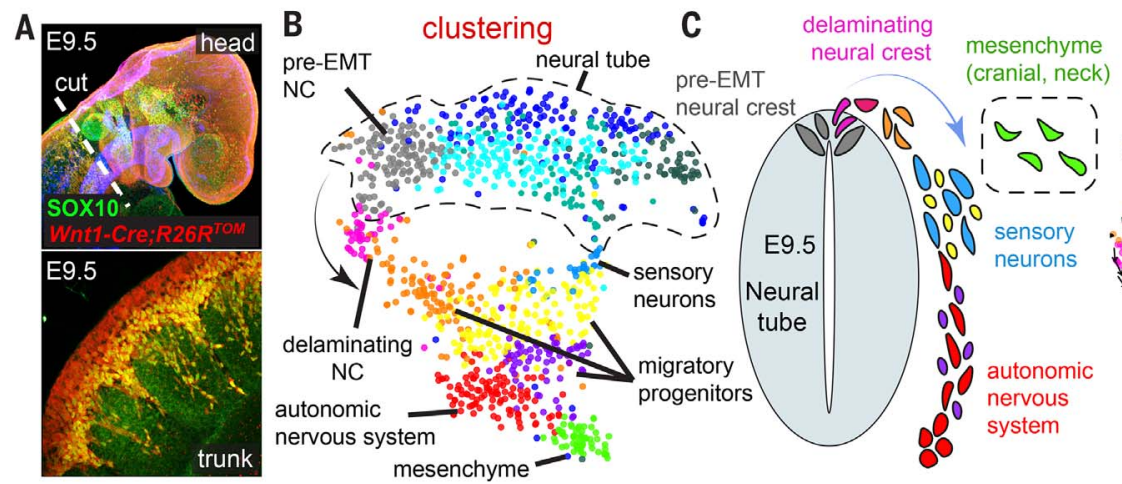

D RNA velocity

E NT NC states F

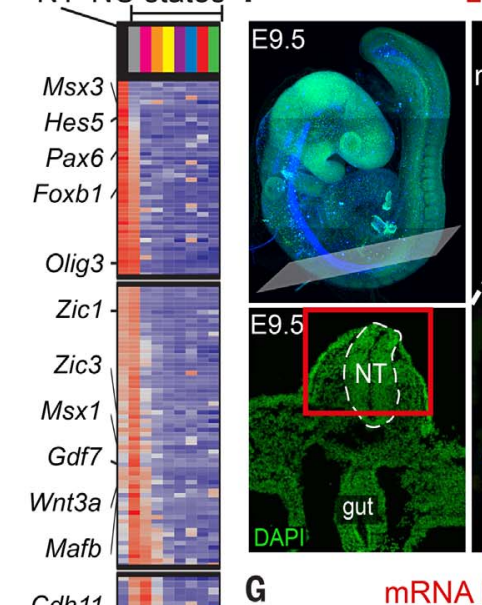

Localization of identified clusters by in situ sequencing

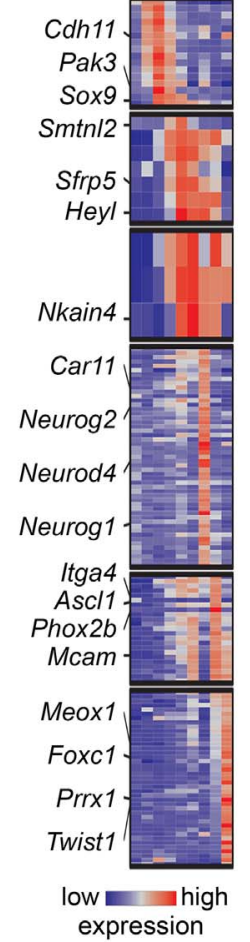

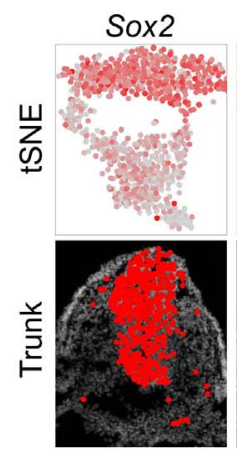
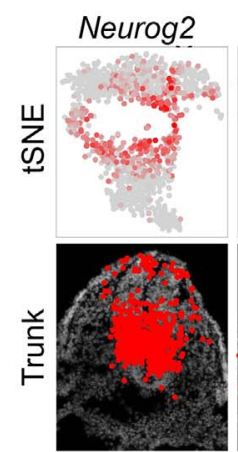

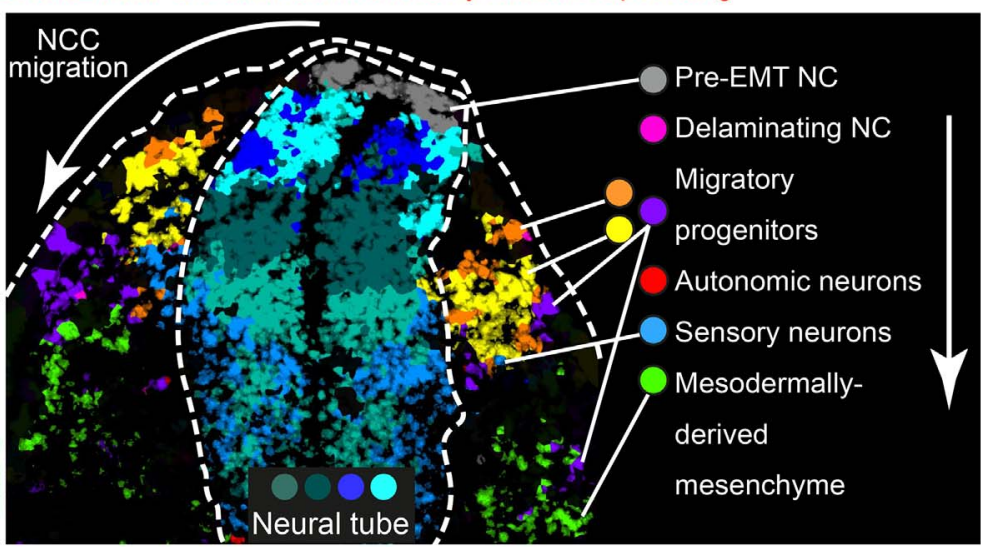

H

Msx1

Gdf7
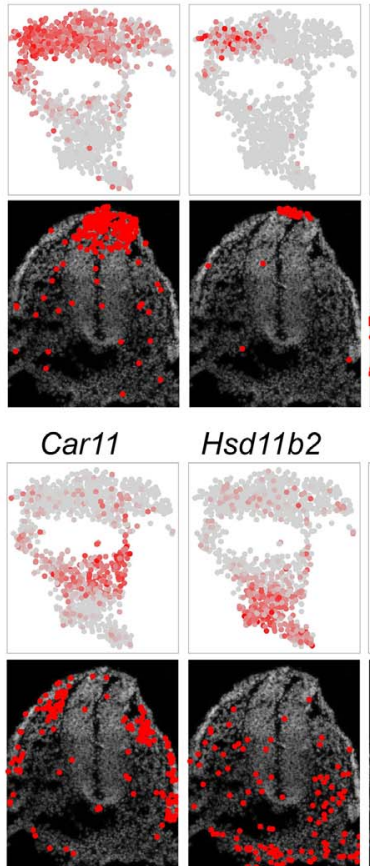
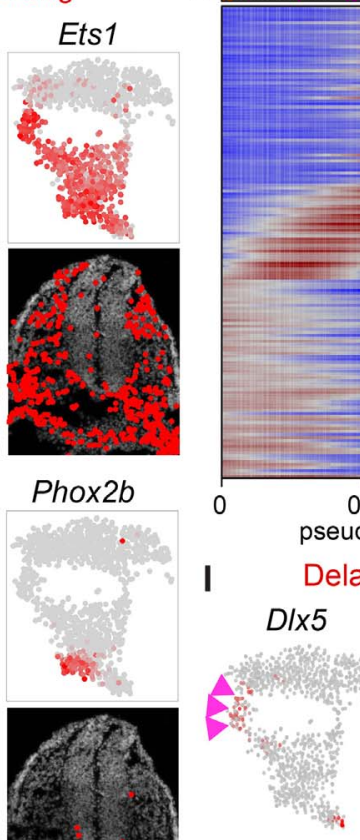

low high

expression 
cells that express Cdh11 or Itga4, genes involved in neural crest migration $(15,16)$, but do not yet express known markers of downstream neural crest fates (Fig. 1E). We will refer to these populations as migrating progenitors. Overall, singlecell data identify substages within the pre- and postmigratory neural crest stages $(6,9)$ and yield sets of marker genes for various stages (table S1).

\section{In situ sequencing shows spatial segregation of distinct neural crest states}

Transcriptional profiles reveal a cascade of states from neural crest formation to fate commitment (Fig. 1, A to E). The early pre-EMT and delaminating crest gives rise to a pool of migrating progenitors, whose heterogeneity is linked to transcriptional properties of the downstream fates (fig. S1D), as they begin to express fatespecific genes. RNA velocity (Fig. 1D) shows a general flow of neural crest cells along the sequence of these developmental stages, followed by divergent flows of migrating progenitors toward more mature neural crest derivatives, including autonomic and sensory neuronal lineage and mesenchyme (Fig. 1, H and I, and data S1). All subpopulations demonstrated robust and uniform cell cycle signatures, except for a small number of cells committed to neuronal lineages (fig. S4, A to D). Immunohistochemistry-validated markers of major subpopulations (fig. S1, F to I) and RNAscope confirm migratory spatial patterns of classical lineage markers and new genes predicted to be implicated in fate specification (fig. S2 and data $\mathrm{S} 12)(17,18)$.

To establish the relative anatomical distribution of the identified subpopulations, we used in situ sequencing (19) to simultaneously detect 32 subpopulation-specific transcripts in a spatially resolved manner in 15 serial sections of the E9.5 embryo (Fig. 1G and data S1 and S11). These two-dimensional measurements confirmed the expected anatomical separation of the neural crest and neural tube populations, and a dorsal neural tube localization of pre-EMT cluster, as well as spatial segregation of mature fates along the dorsoventral axis (Fig. 1, F and G, and fig. S1E). The subpopulations of migratory progenitors showed dorsoventral spatial segregation, with progenitors transcriptionally adjacent to the sensory fate (yellow, Fig. 1B) biased toward dorsal regions, and the progenitors transcriptionally adjacent to autonomic and mesenchymal fates (violet, Fig. 1B) biased toward ventral regions (Fig. 1F and fig. S1E).

\section{Differentiating neural crest undergoes sequential binary fate restrictions}

To disentangle the transcriptional logic of neural crest differentiation in the trunk, we used a branching process to computationally model the progression of emigrating neural crest cells through the heterogeneous pool of progenitors toward committed fates. Specifically, we adapted a principal graph approach (20) to derive a statistical ensemble of contiguous tree trajectories that best explain the observed cell distribution in a high-dimensional transcriptional space (Fig. 2A and fig. S3, A to D; see methods). The resulting trees capture transcriptional changes associated with transitions between pre-EMT, delaminating, and migratory states, followed by multiple cell fate decision branches (figs. S3, A to C, and S4, H to J, and table S3). The developmental potential of neural crest cells has been the subject of previous investigations, showing that preEMT and early migrating neural crest cells are multipotent $(21,22)$. However, it remains unclear how neural crest cells lose multipotency and whether the choice of multiple fates occurs in a stochastic manner or follows a structured pattern of lineage restrictions $(23,24)$. Our results demonstrate a well-defined transcriptional structure of cell-fate splits, which appear as a sequence of binary bifurcations separated by additional robust transcriptional changes (Fig. 2A and figs. $\mathrm{S} 3, \mathrm{~A}$ to $\mathrm{C}$, and $\mathrm{S} 4, \mathrm{H}$ to J). The first stable bifurcation separates sensory lineage from common progenitors of autonomic and mesenchymal branches. The second stable fate split, separating autonomic neuronal fate from mesenchymal differentiation, captures a spatially restricted process that takes place within the cervical region. Additional branches can be attributed to glial differentiation as they show expression of early glial markers (Mpz, Fabp7, Zfp488, Plp1, Sox10) and transcriptional signatures of E10.5 Schwann cell precursors (SCPs) (fig. S4, E to G). Furthermore, removal of the top 50 cells associated with SCP development leads to disappearance of these glia-specific branches (fig. S4, H and I). Although the topology relating sensory, autonomic, and mesenchymal branches is stable, the anchoring of the glial branch is uncertain, with some of the trees in the ensemble placing it within the sensory branch, and others positioning it within the autonomic-mesenchyme branch (fig. S4J). This likely reflects both technical uncertainties arising from limited number of such glial precursors, as well as the fact that glial precursors arise in both sensory and autonomic lineages.

Because it has been previously noted that $W n t 1^{C r e}$ line can activate the Wnt1 signaling pathway and induce a developmental phenotype (25), we generated another single-cell snapshot of the E9.5 trunk neural crest, using a different neural crest-specific Cre line $\left(\right.$ Sox10 ${ }^{\text {CreERT2 }} /$ R2 Romato $\left.^{\text {Tom }}\right)$. Transcriptional dynamics, structure of bifurcations, and patterns of markers expression were similar between $\mathrm{Wnt1}^{\mathrm{Cre}}$ and Sox10 $\mathrm{CreERT2}^{\mathrm{Snap}-}$ shots, indicating that the effect of Wnt1 activation in early neural crest is relatively minor (fig. S5, A to I), consistent with observations by others (26). To evaluate the extent of spatiotemporal conservation of transcriptional program in posterior neural crest, we also generated a single-cell snapshot of hindlimb and tail crest at E10.5 using $W_{n t 1}{ }^{\text {Cre }} / R_{2} 6 R^{\text {Tomato }}$ line. The results recapitulate transcriptional dynamics at E9.5 in trunk, revealing more advanced stages of sensory neurogenesis (fig. S5, H and I). These combined results show that upon maturation, trunk NC proceeds through a stereotypical sequence of binary lineagerestriction decisions.

\section{Distinct functions of early and late} Neurog2 expression

Many genes exhibit statistically significant and robust changes along the reconstructed trunk neural crest developmental tree [1048 genes at a false discovery rate (FDR) of 0.05; Fig. 2, B and C, and table S4], which we grouped into 21 major clusters based on the similarity of their expression patterns (Fig. 2C and data S2). Although expression of many clusters recapitulated the canonical stages of neural crest (6), others captured more complex regulatory patterns, such as down-regulation after delamination followed by reactivation upon mesenchymal specification (cluster 20, data S2). Among the tree-associated genes, we identified $137 \mathrm{TFs}$, many of which were not previously described in the context of neural crest development (e.g., Maf, Ikzf2, Rfxu, Ldb2, Plagl1, Nhlh2) (data S2). Differential expression of a TF, however, does not establish its regulatory role, as TF activity may be modulated by cofactors or other regulatory machinery. We therefore looked for coordinated up-regulation or down-regulation of the predicted $\mathrm{TF}$ targets as an indicator of TF regulatory activity (Fig. 2D). We used regularized linear models (27) to analyze 50 of out 137 trajectory-associated TFs that had known nonredundant sequence binding sequence motifs, and identified TFs whose transcriptional changes show statistically significant regulatory impact on their corresponding target genes (FDR < 0.25) (Fig. 2D and fig. S6). A pattern of TF activity matching TF expression was observed for transcriptional activators, and a complementary pattern for repressors (e.g., Foxd3). A repressive effect of Foxd 3 in non-ectomesenchymal developmental stages is in agreement with the results of FoxD3 loss-of-function experiments (28), including a biasing role of FoxD3 against the mesenchymal program (29). Several TFs showed expression in parts of the neural crest development tree without exhibiting a detectable regulatory impact on their target genes, suggesting modulation by other processes. For example, Insm 1 is expressed in both autonomic and sensory branches, but shows detectable regulatory impact only in the autonomic branch. Indeed, activity of InsmI is modulated by autonomicspecific factor Ascl1 in specification of autonomic lineage (30).

Proneurogenic Neurog2 exhibits two peaks of expression (Fig. 2B): early after the delamination stage that appears to lack direct regulatory impact (Fig. 2E), and late at the onset of sensory neurogenesis that can be linked to corresponding regulatory activity (31). Expression of Neurog2 around the dorsal neural tube has been previously assumed to mark sensory progenitors (32), and in that regard, the early expression of Neurog 2 that precedes any bifurcation points is surprising. It suggests that instead of being limited to sensory lineage, all neural crest derivatives may exhibit transient expression of Neurog2 during differentiation (Fig. 2E). Indeed, lineage tracing in Neurog $2^{\text {CreERT2/+}} ;$ R2 $26 R^{Y F P}$ mouse line starting from E9.5 confirms that cells expressing Neurog 2 at this early stage end up being widely 
A

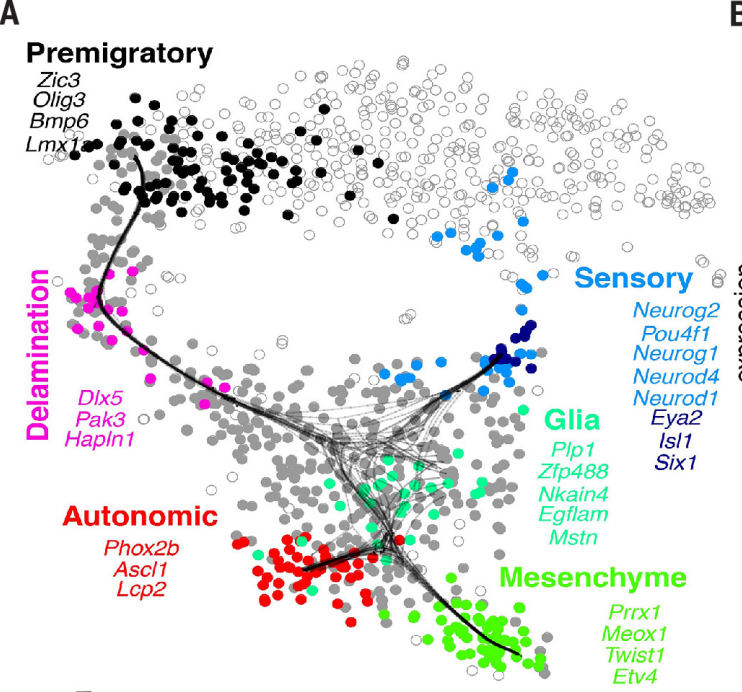

B

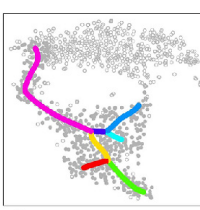

$\downarrow$ fit trajectory

Neurog2

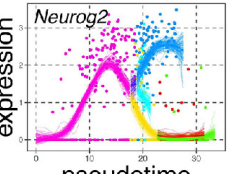

pseudotime $\downarrow$ visualize onto tree

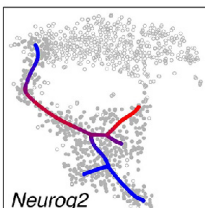

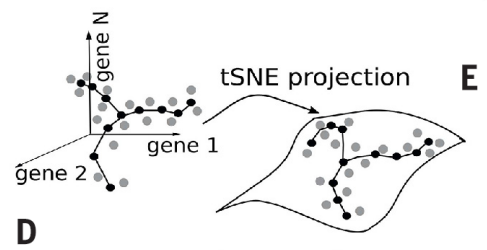

TF target genes cell 1 cell 2 cell 3

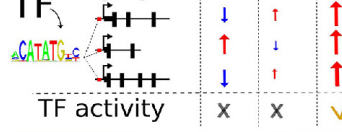

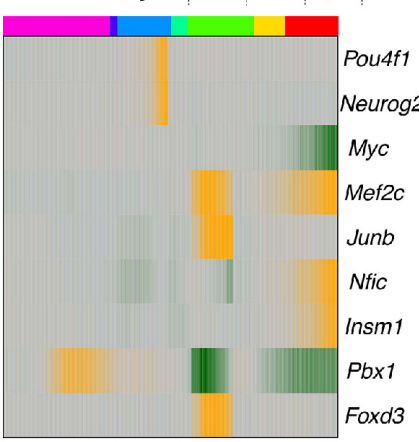

low activity high low expression activity

expression

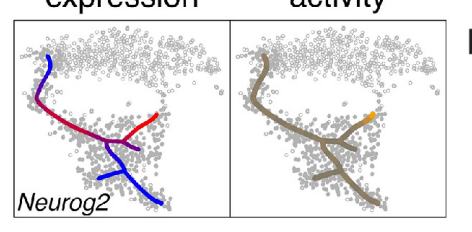

$\mathbf{F}$
C
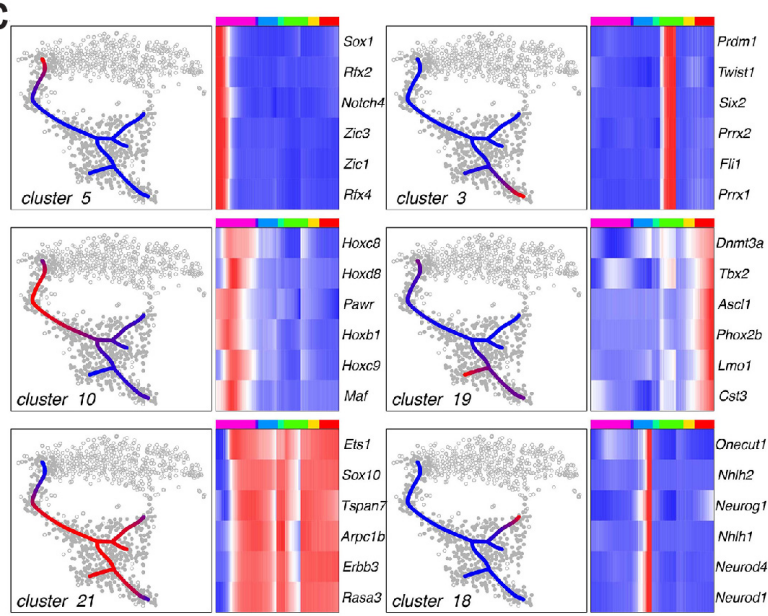

cluster 21

low anigh

expression

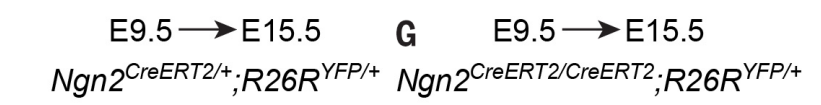
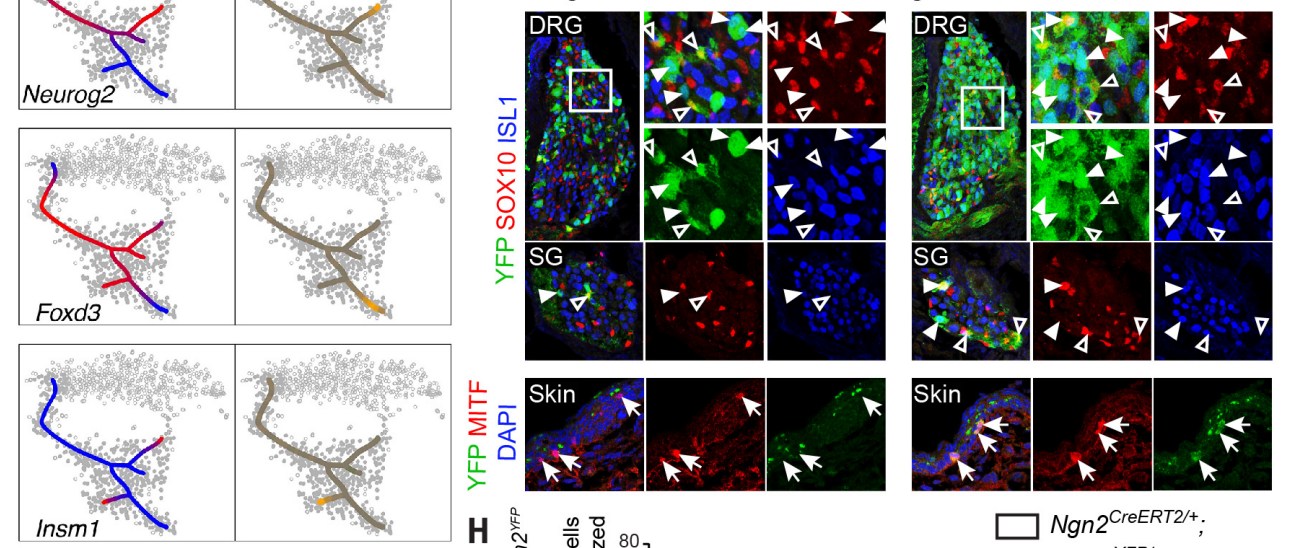

H

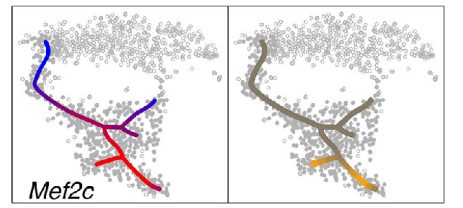

Fig. 2. Developmental portrait of trunk neural crest cells. (A) Developmental progression of trunk NCCs modeled using principal trees. Non-NCCs (empty circles) were excluded from the analysis. Cells with high average expression of fate-specific markers are shown by distinct vibrant colors; a heterogeneous pool of migrating progenitors is shown in gray. (B) Projections of cells onto the principal tree yield smoothed pseudotimeassociated gene expression profiles. Top: Color-coded segments of the principal tree; segment colors match the colors of corresponding unbiased clusters in Fig. 1A, where possible. (C) Clustering of genes based on similarity of NCC expression profiles (see data S1 for extended set of clusters). (D) Transcription factor (TF) regulatory activity along the NCC developmental progression. Lower: A pseudotime activity pattern is shown for a set of TFs predicted to be active during NCC differentiation
(FDR < 0.25). (E) Patterns of expression (left) and activity (right) shown for representative TFs. ( $\mathbf{F}$ and $\mathbf{G}$ ) Immunohistochemistry of control Neurog $2^{\text {CreERT2/+ }} ; R 26 R^{Y F P /+}$ (carrying one copy of CreERT2) and mutant Neurog2 $2^{\text {CreERT2/CreERT2; }}$ R26R ${ }^{\text {YFP/+ }}$ (carrying two copies of CreERT2, see methods for details) tamoxifen-injected at E9.5 and analyzed at E15.5 shows fate partitioning of traced cells between sensory neurons $\left(\mathrm{ISL}^{+}\right.$) (white solid arrowheads), glia (SOX10+) (empty arrowheads), and melanocytes (MITF${ }^{+}$) (white solid arrows). Note that no melanocytes are traced in control embryos. (H) Quantification of the percentage of traced sensory neurons, glia, and melanocytes between control and mutant embryos per organ shows doubling of tracing in neurons and glia, but significantly higher proportion in melanocytes $\left({ }^{*} p<0.05\right.$, ${ }^{* *} p<0.01$, $\left.{ }^{* * *} p<0.001\right)$. DRG, dorsal root ganglion; SG, sympathetic ganglion. distributed across all types of the neural crest progeny in the trunk and are not limited to the canonical sensory fate (Fig. 2F). Earlier studies have shown that Neurog 2 knockout results in transient delay of sensory lineage differentia- tion, which is subsequently compensated by its homolog Neurog1 (31) that also has detectable regulatory impact in the sensory branch (fig. S6A). To assess broader functional impact of early Neurog2 expression on fates distribu- tion, we analyzed knockin Neurog $2^{\text {CreERT2/CreERT2; }}$ $R 26 R^{Y F P}$ embryos (33). In the absence of Neurog2, the efficiency of genetic tracing appeared proportionally higher in all neural crest derivatives except melanocytes, indicating that early 
Neurog2 expression is not primarily associated with committed or biased sensory progenitors. Rather, it suggests a nonneurogenic role of Neurog2 in active repression of the melanocyte fate during early neural crest migration (Fig. 2, G and H).

\section{Coactivation of alternative programs and biasing precede fate commitment}

To investigate the process of cell fate commitment, we examined how transcriptional modules associated with alternative cell fates assemble and compete around decision points, focusing initially on the first bifurcation separating sensory and autonomic branches (Fig. 3A). We approximated fate-specific modules by sets of genes selectively up-regulated in one branch compared to another (Fig. 3, B and C). Whereas some fatespecific genes are up-regulated after the bifurcation point (late genes, 45 sensory, 36 autonomic), others show earlier up-regulation in the progenitor branch, before the actual bifurcation point (early genes, 53 sensory, 86 autonomic) (Fig. 3B and data files S3 and S4). For example, Neurod1, thought to be a master regulator of sensory fate, is induced late in the sensory branch, whereas another sensory TF, Pou4fl, is up-regulated earlier during delamination, in a manner similar to that of Neurog2 (Fig. 3B). This is expected, as bifurcation points capture trajectory positions where the extent of the transcriptional differences between the alternative cell fates becomes sufficiently large. The expression onset differences were also confirmed by the RNAscope measurements of select genes in both sensory and autonomic branches (fig. S2). We observed that early modules of competing cellular fates are coexpressed in progenitor cells and exhibit gradual coactivation as the cells progress toward the bifurcation point, followed by selective upregulation of one module and down-regulation of another after the bifurcation point (Fig. 3C). By contrast, late-competing modules show mutually exclusive activation in their corresponding branches, without coexpression in individual cells, consistent with commitment to a particular fate (Fig. 3D). Many known critical master regulators do not show detectable expression around the bifurcation point (including autonomic Phox 2b, Ascl1, and sensory Neurog1, Neurod4, and Neurod1), indicating contribution of other genes or mechanisms to the early steps of the decision process. These likely include exposure to extracellular signals such as Wnt signaling, BMP signaling, or Delta-Notch interactions (34)

We next examined whether despite general coactivation of competing-fate modules, the progenitor cells exhibit an early transcriptional bias toward a particular fate beyond the effects of expression noise. In doing so, we controlled for apparent biases due to developmental asynchrony of the subpopulations being analyzed, which can limit interpretation of similar analysis based on targeted or bulk measurements (fig. S7I) (2). For the cells positioned around the actual bifurcation point, the transcriptional bias toward one of the possible fates manifests itself as a pronounced negative correlation between the competing gene modules (Fig. 3E). We find that as cells move toward the first bifurcation point following the initial coactivation stage, the degree of transcriptional coordination within each module increases, and antagonistic expression of the competing modules becomes more apparent (Fig. 3, E and F). Simulated controls, with expression profiles randomized across cells with similar pseudotime to preserve the overall gene activation profiles, fail to show such local coordination of gene modules (fig. S7, A and B). This indicates that as cells progress toward the bifurcation point, they undergo gradual transcriptional biasing toward one of the competing fates. The biasing phase does not appear to be driven by up-regulation of a single gene. Rather, we observe broad expression shifts between genes of the competing fate-specific modules (Fig. 3F). These results indicate the presence of lineage priming early in neural crest migration, prior to the predicted sensory-autonomic bifurcation point. The transcriptional correlations within the competing modules are reduced following the bifurcation point. This reduction illustrates that most of the observed intramodule correlation can be attributed to compositional heterogeneity of cells around the bifurcation point (35). When considering more homogeneous cell populations within the branches following the bifurcation point, correlation of genes within the module becomes relatively low, indicating that regulatory interactions between genes, such as correlations induced by stochastic covariation of a TF and its target genes, are not apparent in such data (36) (Fig. 3F).

To determine the initial transcriptional events in the assembly of the fate-specific modules, we monitored coordinated expression of gene subsets, tracking backward along the progenitor branch. In doing so, we controlled for the overall pattern of module activation, as such general trends can overshadow correlations within a subset (fig. S8). The results show that early correlated subsets of modules specific to either sensory or autonomic fate can be statistically distinguished at the delamination stage, but not in the pre-EMT neural crest (Fig. 3G and data files S5 and S6). We detect earlier formation of the sensory module, compared to the autonomic module. Overall, we observe three primary phases of cell fate decision, formalized as a sequence of initial coactivation, gradual biasing, and commitment phases (Fig. $3 \mathrm{H}$ ). The initial coactivation stage is characterized by coexpression of competing modules within individual cells that gradually shifts in favor of one of the modules during the biasing phase, culminating in upregulation of mutually exclusive fate-specific gene expression patterns during commitment. The three-phase scheme of cell fate decision also holds for the autonomic-mesenchyme bifurcation discussed below (fig. S7, C to H). This model elaborates on earlier observations of coexpression of key regulatory factors (37-40) or programs (41-43) from alternative fates prior to cell fate commitment noted in other tissues.

\section{Autonomic-mesenchymal bifurcation reveals bipotent progenitors}

Studies of neuroblastoma, a tumor of sympathetic nervous system that originates from neural crest cells, revealed that tumor cells can transit between adrenergic and mesenchymal phenotypes, creating inter- and intra-tumoral heterogeneity that poses a therapeutic challenge $(44,45)$. Our data uncovered the developmental bifurcation that separates autonomic and mesenchymal fates (Fig. 4A). After the initial stage of co-activation of both programs (Fig. S7C-E), commitment to fates is marked by expression of two fate-specific late genes: Phox $2 b$, a key driver of autonomic neuronal lineage (46); and Prrx1, a marker of specified mesenchyme (47). However, some transient cells co-express both genes at high levels (Fig. 4A). Consistent with this, corresponding Prrx1 and Phox $2 b$ mRNA molecules colocalize and proteins are coexpressed in the same few cells in the ventral neural crest pathway of migration (Fig. 4, C and D). Nearly all late genes of the autonomic module are coexpressed with late genes of the mesenchymal module in at least a few cells, illustrating ubiquitous stochastic coactivation of the alternative fate modules (Fig. 4B).

To test whether the cells expressing Phox $2 b$ can end up in the mesenchymal domain, we performed lineage tracing with a Phox $2 b^{C r e} /$ R26R ${ }^{\text {TOMATO }}$ mouse line (Fig. 4, F and G). As expected, the autonomic neurons and numerous glial cells proximal to autonomic components were traced. However, we also observed tracing of multiple cells of the mesenchymal phenotype in the cervical region, as well as in the heart, in the proximity of autonomic components (parasympathetic neurons of the heart) (Fig. 4H). Detailed analysis at the region of the heart and the proximal autonomic ganglia showed that $21.6 \pm 3.1 \%$ of traced cells contributed to glia, $9.9 \pm 1.6 \%$ to autonomic neurons, and $68.4 \pm 4.7 \%$ to mesenchyme (Fig. $4 \mathrm{H}$ ). These results confirm that some of the cells expressing late-autonomic signatures subsequently deviate toward mesenchymal specification in the cervical region. The opposite experiment with lineage tracing in Prrx1-Cre;R26R ${ }^{m T m G}$ showed no traced autonomic neurons or glial cells in the entire embryo, including the cervical region where the large superior cervical ganglion is positioned (Fig. 4, E and $\mathrm{H}$ ). Thus, cells expressing Prrx1 always differentiate into mesenchymal fates. As a result, when both Prrx1 and Phox $2 b$ master regulators are coactivated in the same cells, cell fate is resolved in the direction of mesenchymal derivates in vivo (Fig. 4B). No sensory neurons were traced from progenitors expressing Phox $2 b$, demonstrating that $P h o x 2 b^{+}$cells at the second bifurcation point are bipotent. The possibility of transdifferentiation of more committed $\mathrm{AsclI}^{+} / \mathrm{Phox}_{2 \mathrm{~b}^{+}}$ autonomic progenitors into mesenchymal population is not supported by RNA velocity analysis (Fig. 1D) and lineage tracing from E10.5 with Ascli $^{\text {CreERT2 }}$;R26R ${ }^{\text {TOM }}$ (fig. S9). 
A

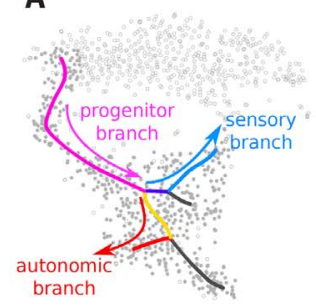

D Co-activation

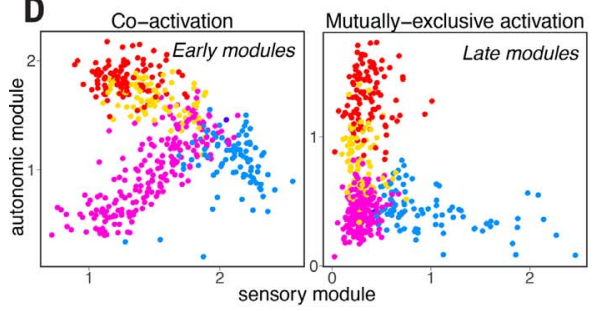

B $\quad$ expression

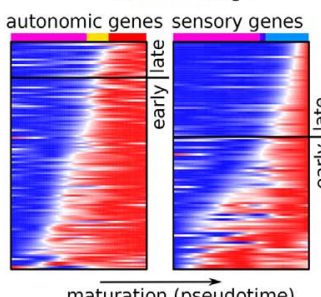

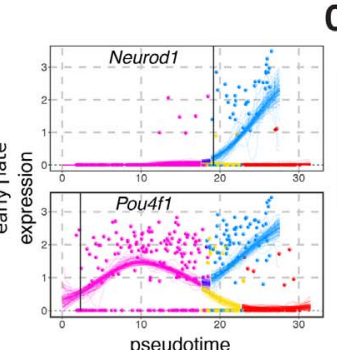

pseudotime
C

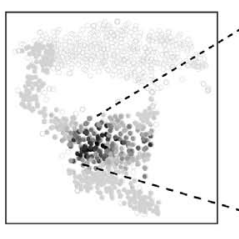

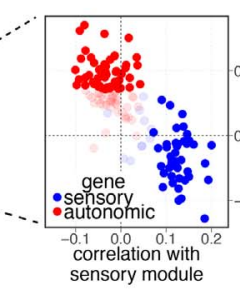

sensory module

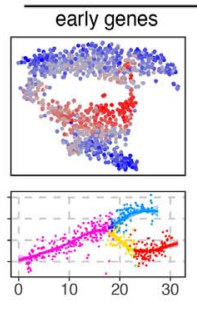

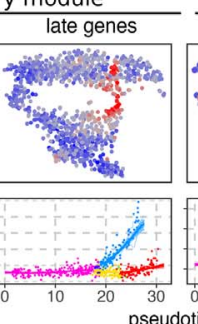

pseudotime

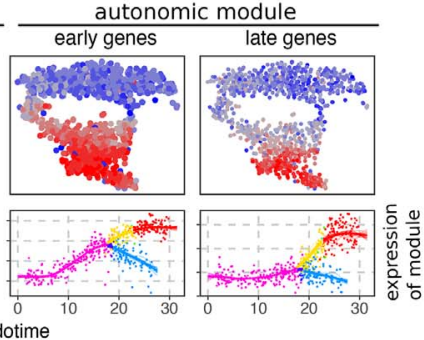

G

F gradual coordination of branch-specific modules

premigratory delaminatory early migratory
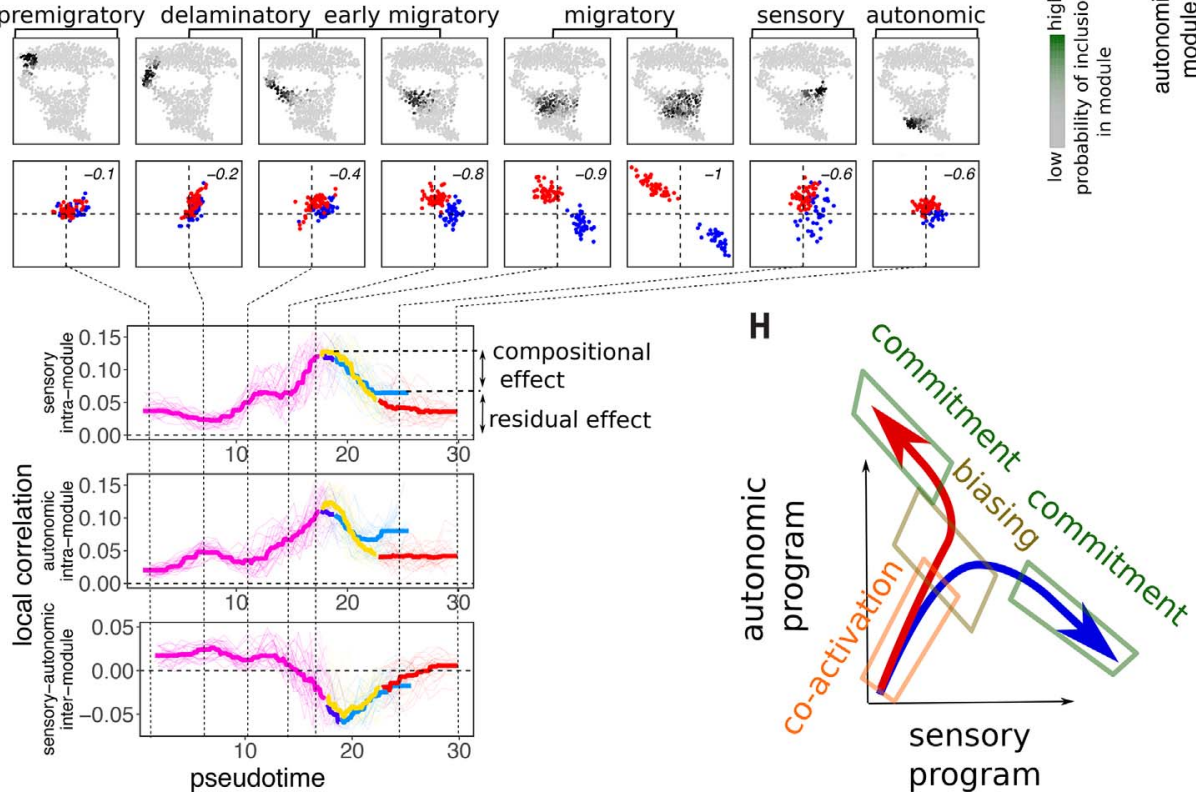

Fig. 3. Logic of cell fate selection for sensory-autonomic bifurcation.

(A) Schematic overview of the sensory-autonomic split in NC

differentiation. (B) Branch-specific (sensory versus autonomic) genes classified as activated early (before split) and late (after split). Right: Example of an early (Pou4f1) and a late (Neurod1) sensory-specific gene. Vertical line marks predicted time of up-regulation. Time of up-regulation of all genes can be found in data S3 and S4. Colors encode branches [as in (A)]. (C) Average expression pattern of early and late branch-specific modules. Early and late modules are set of genes activated before or after the bifurcation. (D) Scatter plots show average expression of sensory and autonomic modules in each cell. Left: Early competing modules show gradual coactivation, followed by selective up-regulation of one fate-specific module and down-regulation of another. Right: Late modules show almost mutually exclusive up-regulation. Colors encode tree branches. (E) Bifurcation point is characterized by correlated expression of genes within modules, and repulsion of competing modules. Left: Probability of a cell proximity to the sensory-autonomic bifurcation point shown (darker colors corresponding to higher probability). Right: Average correlation of each early sensory- or autonomic-specific gene (points) with the genes from the sensory- ( $x$ axis) and autonomic-specific ( $y$ axis) modules among cells around the bifurcation point. Genes that have low average correlation with their own module $(<0.07)$ are shown with faded colors and are excluded from analysis in (F) for clarity. (F) Early modules show gradual intramodule coordination and intermodule repulsion. Upper: The plots show average local correlations of module genes with autonomic and sensory modules in a set of cells with similar developmental time (marked by black points). Bottom: Average local correlations of genes within and between branchspecific modules show gradual increase in coordination within each module and increasing antagonistic expression between the branchspecific modules. The difference between intra- and intermodule correlations, which characterize the extent of the antagonistic expression between the alternate modules, is shown in the upper right corner of tSNEs. (G) Time of gene inclusion in coordinated branch-specific module shows early signatures of fate-biased expression programs. Bottom: Schematic illustrating of formation of a branch-specific module, with connecting lines indicating onset of expression correlation between genes. Heatmap: Probabilities of gene inclusion in the branch-specific module during early stages of differentiation (see data S5 and S6 for gene names). Right: Examples of inclusion probability estimates for individual genes in the two modules. (H) Cell fate decisions formalized as a three-phase process: Initially, cells coactivate competing cell fate-specific programs, gradually switch to preferential expression of one module, and culminate by activating mutually exclusive fate-specific expression. 
A

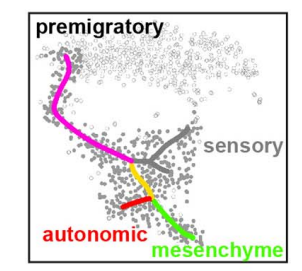

Late modules:

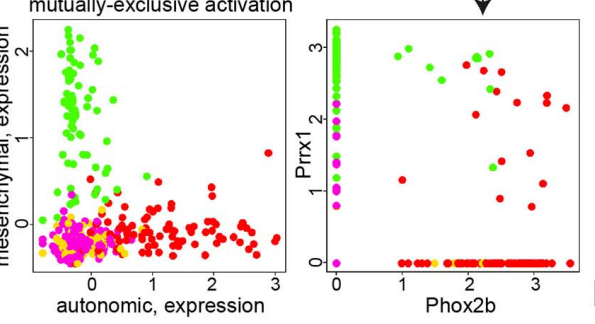

B

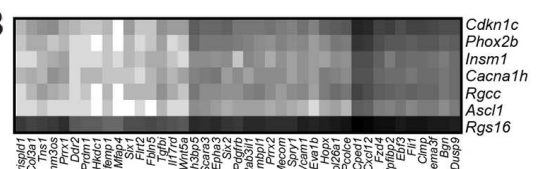

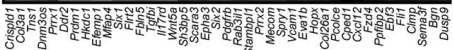
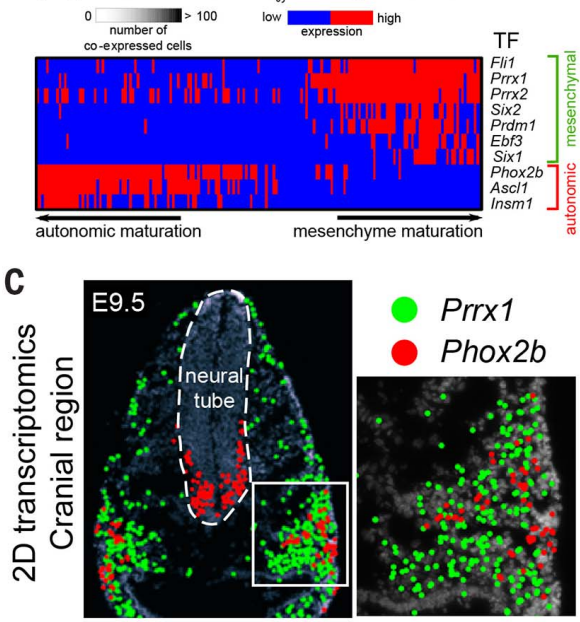

D PHOX2B SOX10 PRX1MERGEPHOX2BPRX1

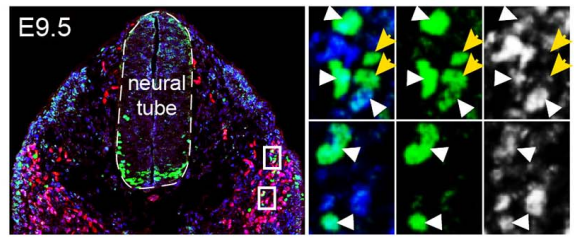

Fig. 4. Phox $2 b$ and Prrx1 coexpression reveals bipotent progenitors.

(A) Infrequent coexpression of Phox $2 b$ and Prrx1 is observed despite mutually exclusive expression of modules. Bottom left: Late modules of the autonomic-mesenchyme bifurcation show mutually exclusive expression. Bottom right: Prrx 1 and Phox $2 b$ show largely branch-specific expression pattern, but are coexpressed in some cells. (B) The majority of antagonistic pairs of branch-specifying genes are coexpressed at high levels in a few cells. Upper: Heatmap with color indicating numbers of cells with coexpression of two genes from competing late modules (rows, autonomic; columns, mesenchymal). Lower: Heatmap showing expression of branch-specific TFs in cells assigned to either branch. (C) Pattern of Prrx 1 and Phox $2 b$ expression (by in situ sequencing) at E9.5. Coexpression of the two genes is observed at the prospective cranial ganglia.

(D) Immunofluorescence for PHOX2B, PRX1, and SOX10 in E9.5 trunk

( $\mathrm{PHOX} 2 \mathrm{~B}^{+} / \mathrm{PRX} 1^{+}$cells shown by white arrowheads, $\mathrm{PHOX}_{2} \mathrm{~B}^{+} / \mathrm{PRX}^{-}$by
E E17.5 Prrx1 ${ }^{\text {GFP }}$ S100 ISL1

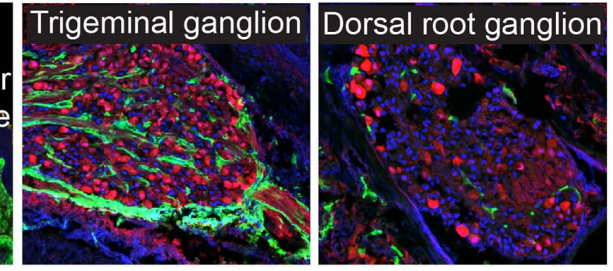

E17.5 Prrx ${ }^{\text {GFP }}$ S100 ISL1 E17.5 Prrx ${ }^{\text {GFP ISL1 COLIV }}$

Superior cervical

ganglion
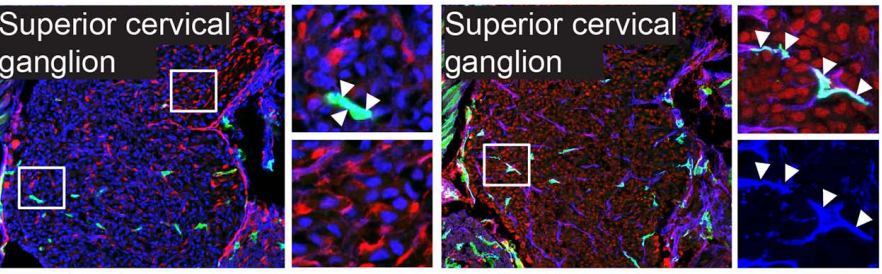

E13.5 heart

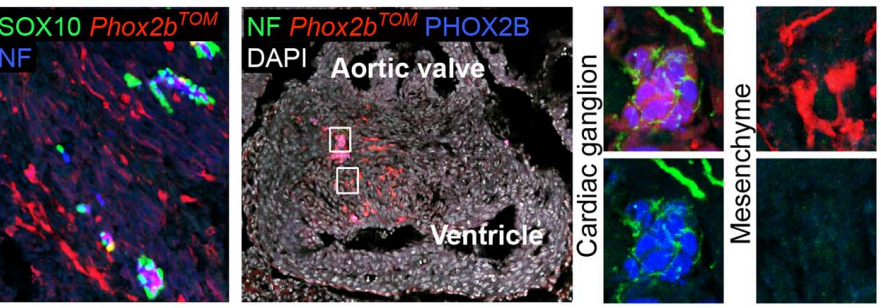

E13.5 heart
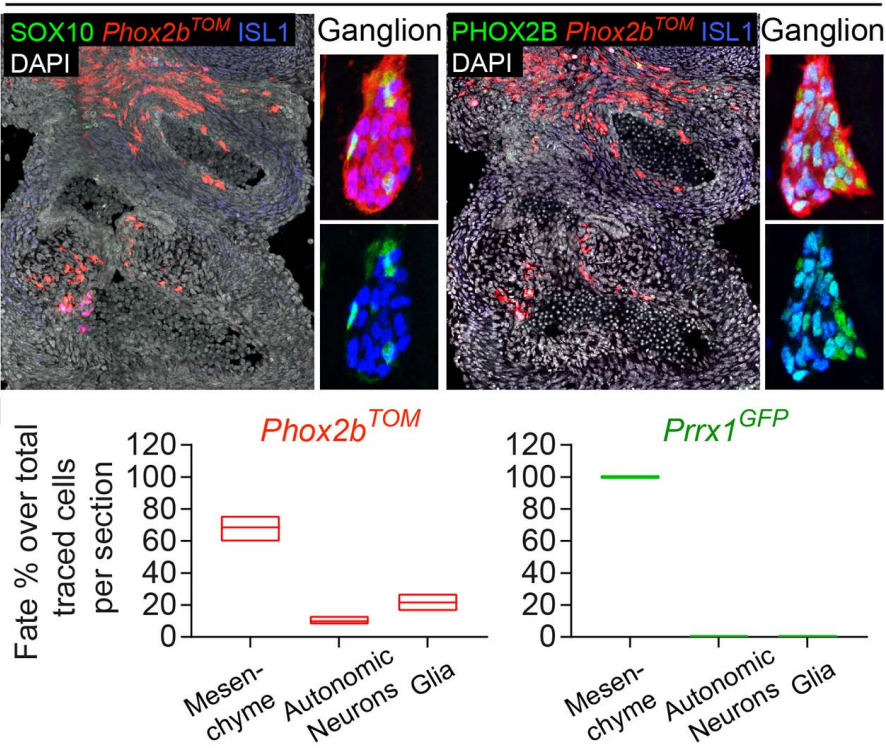

yellow arrows). (E) Immunohistochemistry on E17.5 embryo for Prrx ${ }^{\text {GFP }}$, S100, and ISL1 shows absence of overlap between the mesenchyme $\left(\operatorname{Prr} x 1^{G F P+}\right)$, glia, and sensory neurons. Note that Prrx $1^{\text {GFP+ }}$ shows colocalization with COLIV, indicating that the vascular mesenchyme was traced. (F) Immunohistochemistry on E13.5 embryo for SOX10, Phox $2 b^{\text {TOM+ }}$, neurofilaments, and PHOX2B (in heart-related panels). Note the presence of numerous Phox $2 b^{T O M+} / \mathrm{PHOX}_{2} \mathrm{~B}^{-}$mesenchymal cells in the cervical region and surrounding Phox $2 b^{T O M+} / \mathrm{PHOX}_{2} \mathrm{~B}^{+}$ autonomic neurons in the heart. (G) Immunohistochemistry on E13.5 embryo for SOX10 or PHOX2B, Phox $2 b^{T O M+}$, and ISL1. Note the presence of numerous Phox $2 b^{T O M+} /$ mesenchymal cells in aortic areas, negative for ISL1, PHOX2B, or SOX10. (H) Analysis of the percentage contribution of Phox $2 b^{T O M+}$ and Prrx ${ }^{G F P+}$ cells to mesenchyme, autonomic neurons, and glia over the total number of traced cells in the area of the heart ( $N=3$ embryos per strain). 


\section{Transcriptional specificity of cranial neural crest emerges during delamination}

Migration and differentiation of cranial neural crest are spatiotemporally separated from events occurring in the trunk. The cranial crest primarily gives rise to mesenchymal populations responsible for building the head, which are not produced by the trunk crest (48). We analyzed single-cell snapshots of anterior cranial, pre-otic, and postotic Wnt1-traced populations at E8.5 (Fig. 5A). Early neural crest was composed of three spatially distinct populations: $\mathrm{Hox}^{-}$population that corresponds to the anterior cranial neural crest, $\mathrm{Hoxb}_{2}{ }^{+}$that contributes primarily to the mandibular level, and $\mathrm{HoxD}^{+}$population that includes post-otic (including cardiac and vagal) streams of the neural crest (Fig. 5A, fig. S10A, table S5, and data S7) (49). The developmental portrait of $H_{0 x}{ }^{-}$anterior cranial neural crest at E8.5 represented a single differentiation trajectory that encompasses diverse transcriptional events: down-regulation of a neural tube-associated program, including Zic3 and Pax8; transient expression of a battery of genes such as Pak3 and Bmper; coherent, but not simultaneous, up-regulation of neural crest markers, including Foxd3, Sox 9 and Sox10, and mesenchymal fate-specifying genes, including the TFs Twist1 and Prrx2 (Fig. 5, B and C, table S6, and data S8). Consistent with this, analysis of $H_{0 x}$ neural crest variability shows trajectory-associated and cell cycle processes (fig. S10G). Although we did not observe fate bifurcations in the cranial crest at the E8.5 stage, an anticorrelation of the mesenchymal and neuronal genetic programs emerged, coinciding with the down-regulation of the early neural crest genes Foxd3 and Sox 9 and activation of the mesenchymal factors (Fig. 5D and fig. S10B). Thus, cranial neural crest, similar to trunk cells, progresses through a biasing stage before commitment to a specific fate. This indicates that signs of ongoing cell fate bifurcations often can be detected in advance, similar to early detection of transitions noted in other fields (50). As in the case of the anterior cranial neural crest, the snapshot of $H_{o x} b 2^{+}$neural crest cells underwent a differentiation progression through an intermediate step toward migrating cells expressing mesenchymal markers (fig. S10, C and D; Table S7; and data S9). Hoxb2 $2^{-} /$Hoxd $^{+}$neural crest population was represented as an isolated cluster.

Cranial neural crest at all spatial levels expresses a shared core program (including classical markers Sox10, Sox 9, Foxd3, and Ets1). As a result, $\mathrm{Hox}^{-}$ and $\mathrm{Hoxb2}^{+}$neural crest subpopulations are more similar to each other than to the corresponding neural tube regions from which they originate (Fig. 5E). Nevertheless, $H_{0 x}{ }^{-}$and $H o x b 2^{+}$neural crest shows expression differences (fig. S10, E and F), which may be a consequence of divergent differentiation trajectories, or simply reflect residual differences of their distinct starting states prior to delamination from the neural tube. We find evidence for the former, with the difference between $\mathrm{Hox}^{-}$and $\mathrm{Hoxb}_{2}{ }^{+}$neural crest correlating well with the differences in the neural crest developmental programs induced at the corresponding spatial levels $(r=0.49)$, but not correlated with the difference of their starting $\mathrm{Hox}^{-}$ and $\mathrm{Hoxb}_{2}{ }^{+}$neural tube states (Fig. 5E). This indicates that the transcriptional divergence of the neural crest from different spatial levels arises primarily during downstream delamination and migration stages through the activation of new gene expression programs.

\section{Cranial and trunk crest follow different paths through a shared differentiation landscape}

To compare more-differentiated populations arising from different neural crest levels, we further sampled crest-derived cells of cranial region at E9.5, post-otic (vagal and cardiac) at E10.5, and lumbar and tail regions at E10.5. Joint alignment of all crest datasets (see methods) revealed that neural crest cells at various positional levels and time points navigate a stereotypic space of transcriptional states, with some notable biases (Fig. 5F; fig. S10, H and I; and tables S9 and S10). The distribution of mature cells of the cranial compartment is biased toward mesenchymal fate, whereas cells of the trunk compartments gravitate toward sensory and autonomic fates (Fig. 5G and fig. S10M). In line with this, the fraction of mesenchymal committed cells was high in the anterior population, whereas the fraction of sensory committed cells was high in the posterior population as inferred from $H o x$ gene expression (fig. S10J). The cranial neural crest progressed to a more mature mesenchymal state at E9.5 as compared to the earlier E8.5 time point, as evident from activation of Prrx 1 and Twist2. By contrast, trunk neural crest showed active maturation of sensory progenitors from E9.5 to E10.5, including activation of Dcx,Stmn2, and down-regulation of Neurog2 (fig. S10I). Postotic neural crest occupied an intermediate position between anterior cranial and trunk neural crest, and shared some features with each of those populations (51). Snapshot of post-otic neural crest populations at E10.5 represented mostly Hoxd $3^{+}$cells, indicating a dominant contribution from the vagal neural crest (Fig. 5G). The population contains cells of autonomic and cardiac-mesenchyme fates with a small admixture of sensory-committed cells. Cardiac mesenchyme is necessary for development of cardiac valves and the outflow tract (52). Heterogeneity of this subpopulation revealed three initial steps of ectomesenchyme differentiation that are marked by sequential acquisition and downregulation of Six 2 followed by transient upregulation of $D l x 6$ and activation of $M s x 2$ and heart-specific Hand2, and then activation of downstream cardiac markers Hand1, Dkk1, and Gata6 (Fig. 5H, table S8, and data S10). Screening of the neural crest snapshot at E8.5, E9.5, and E10.5 stages did not reveal coexpression of the melanoblast markers (Mitf, Pmel, Dct) or a broader transcriptional signature of prospective melanoblasts at any of the spatiotemporal levels, indicating late consolidation of melanocytic fate (fig. S10, $\mathrm{K}$ and L).
During delamination and early migration stages, both cranial and trunk neural crest progress through a similar sequence of transcriptional steps, as illustrated by intermixing in joint expression space (Fig. 5G and fig. S11A). Nevertheless, in addition to such common maturation signatures, cranial and trunk neural crest also activate their own distinct modules (Fig. 6A and fig. S11, A and D). For cranial cells, this includes activation of a broad mesenchymal program, including regulatory factors such as Twist1, Prrx2, and Dlx2 (Fig. 6B), whereas activation of the sensory program that includes Neurog2, Pou4f1, and Nkx2.1 is specific to trunk cells. Therefore, we hypothesized that early activation of fate-specific programs after delamination can predispose cells toward specific fates and account for the distinct downstream fate biases of trunk and cranial neural crest cells.

\section{Twist1 activation is sufficient to reroute trunk neural crest into a mesenchymal fate}

To test if activation of mesenchymal regulators can bias trunk neural crest toward mesenchymal differentiation, we electroporated Twist1expression constructs into the developing chick neural crest. Such targeted and sustained overexpression of Twist 1 in the trunk neural crest starting from pre-EMT stage resulted in downregulation of traditional trunk fates such as neuronal sensory, autonomic, and glial and alteration of the neural crest migration patterns (Fig. 6C). Instead of the expected migration toward the dorsal aorta, Twist $1^{+}$cells in the trunk predominantly followed a path to dermal regions, where they activated expression of a dermal mesenchymal marker Prrx1, not observed in wild-type trunk neural crest (Fig. 6C). Some Twist1 ${ }^{+}$cells migrated to sensory ganglia and the dorsal aorta, where they did not take neuronal or glial fates (fig. S12, A and B). Conversely, CRISPR-Cas9based knockdown of Twist1 in chick cranial neural crest resulted in a reduction of mesenchymal populations and an increase in glial and neuronal derivatives (fig. S12, C to F), consistent with previous studies $(53,54)$.

Overexpression of the downstream mesenchymal factor Prrx 2 in the trunk resulted in massive apoptosis of neural crest cells (fig. S11B). The rare surviving cells were not able to pick traditional trunk fates, as compared to controls [green fluorescent protein (GFP)-only DNA or neuro-glia-related genes, such as Crabp1; fig. S11B]. By contrast, regulator of sensory fate Pou4fI is strongly activated upon delamination in the trunk, but is poorly expressed in cranial crest: Overexpression of the dominant-negative version of Brn3c, a homolog of Pou $4 f 1$ in chick, in cranial and trunk chick neural crest resulted in no obvious phenotype in the trunk, as expected, whereas it caused a decrease of $\operatorname{Sox} 9^{+} / \mathrm{Pax}^{+}$ cranial neural crest cells (fig. S11C). This suggests that Pou 4 f1 might be another factor differentially regulating fates in different $\mathrm{NC}$ populations along the anteroposterior axis. 
Fig. 5. Spatiotemporal analysis of cranial neural crest. (A) t-SNE embedding shows subpopulations observed in 1345 cells collected from the cranial part of E8.5 Wnt1-Cre:R26 ${ }^{\text {TOM+ }}$ embryos (left: representative embryo shown). (B) Analysis of the developmental progression identifies timing of transcriptional events of early $\mathrm{Hox}^{-}$cranial NCCs. (C) Pseudotime expression trends of representative genes [from (B)] are shown for early $\mathrm{Hox}^{-}$cranial NCCs.

(D) Correlation of

fate-specific gene modules reveals emergence of mesenchymal versus neuronal biases in migration. Similar to Fig. 3F, the cells were arranged by pseudotime and binned in groups of 50 cells (upper panels) to estimate intra- and intermodule correlations between the mesenchymal and neuronal (sensory, autonomic) gene modules from E9.5 trunk NCC analysis (lower panels). (E) Transcriptional differences of migratory $\mathrm{Hoxb}_{2}{ }^{+}$ and $\mathrm{Hox}^{-}$cranial NCCs emerge during delamination. Left: NCCs from Hox- and $\mathrm{Hoxb}^{+}$populations show more similar profiles in the migratory stage, compared to NT, whereas transcriptional differences between Hox- and $\mathrm{Hoxb2}^{+}$in NT $(\Delta \mathrm{NT})$ and migratory stage $(\Delta \mathrm{NC})$ are not correlated. Right: The difference in transcriptional shifts accompa-

nying delamination in $\mathrm{Hoxb2}^{+}\left(\mathrm{dNC}^{+}\right)$and Hox- $\left(\mathrm{dNC}^{-}\right)$NCCs correlates with the resulting expression difference between $\mathrm{Hox}^{-}$and $\mathrm{Hoxb2}^{+}$populations at the migratory stage $(\Delta \mathrm{NC})$, indicating that most migratory differences between levels emerge during delamination, instead of reflecting initial differences at the NTstage. (F) A joint embedding of NCCs from different stages (E8.5, E9.5, E10.5) and locations (cranial, trunk, cardiac, hindlimb, and tail) shows stereotypic landscape of NC development. Inset shows schematic tree of differentiation. (G) Distribution of transcriptional states of spatiotemporally distinct datasets (colored according to developmental time). (H) Three early ectomesenchyme developmental states of progressive maturation in cardiac NC population.
A

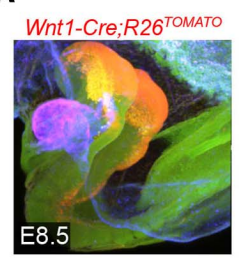

B

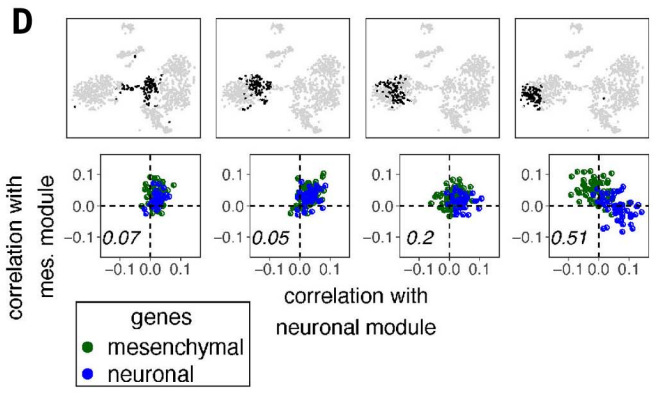

$\mathbf{F}$

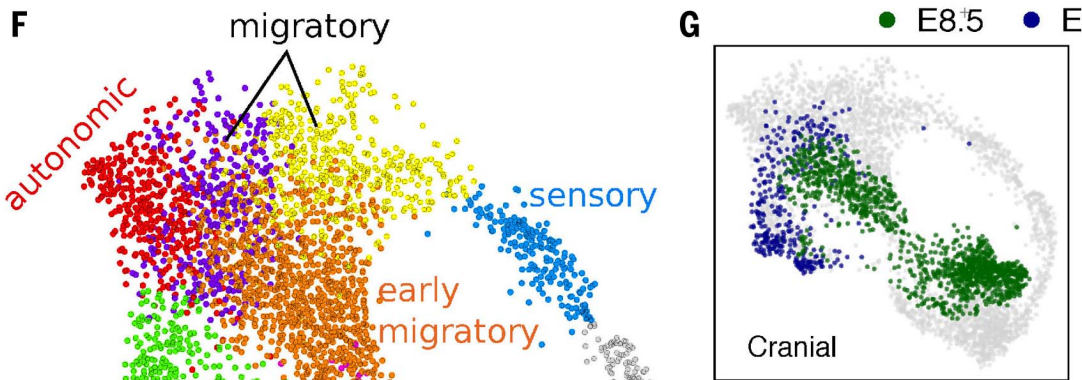

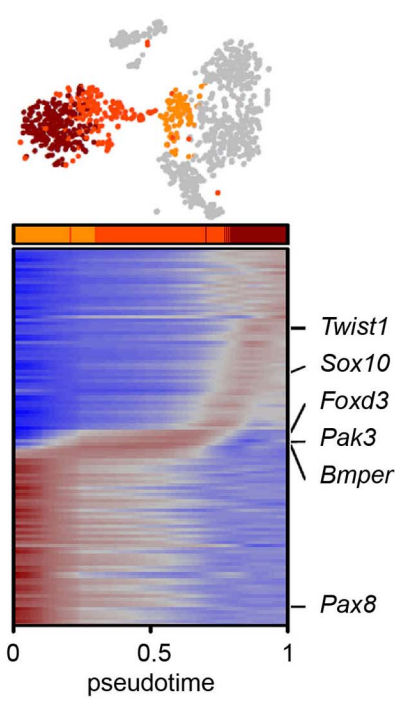

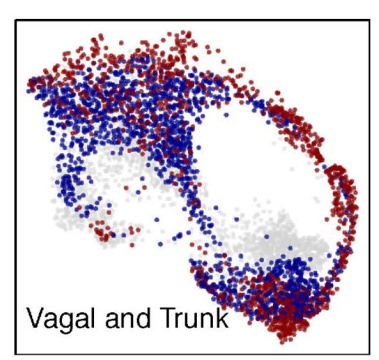

C
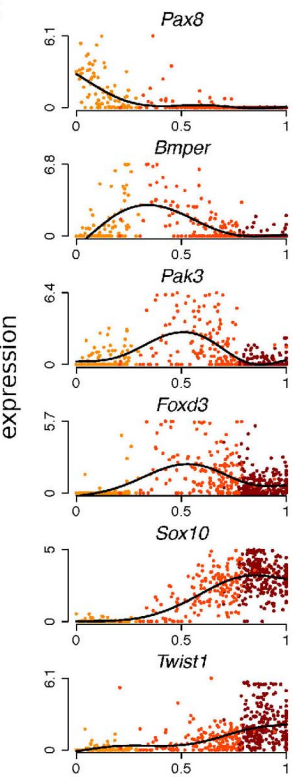

pseudotime
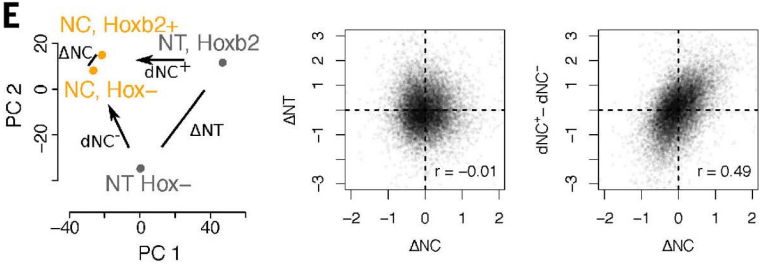

E10.5
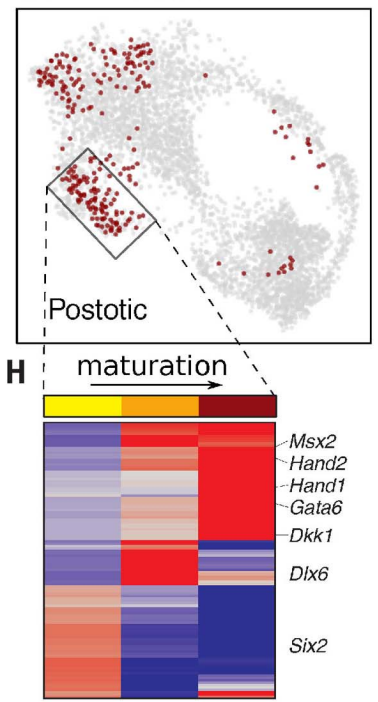
A

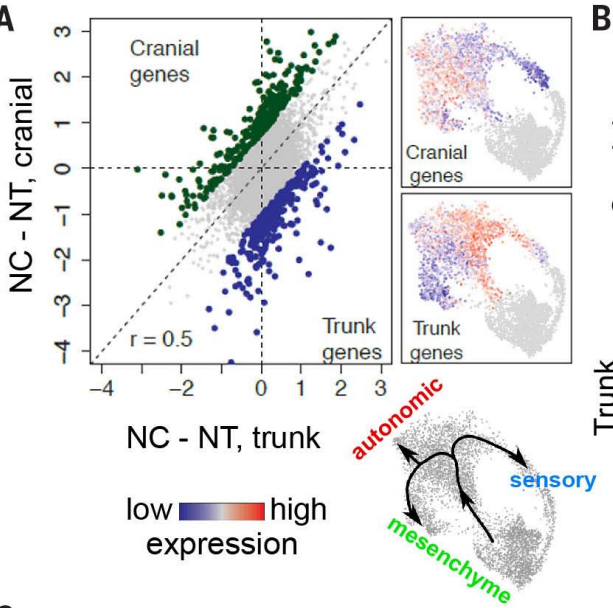

C Electroporation of control RFP plasmid into trunk NCC

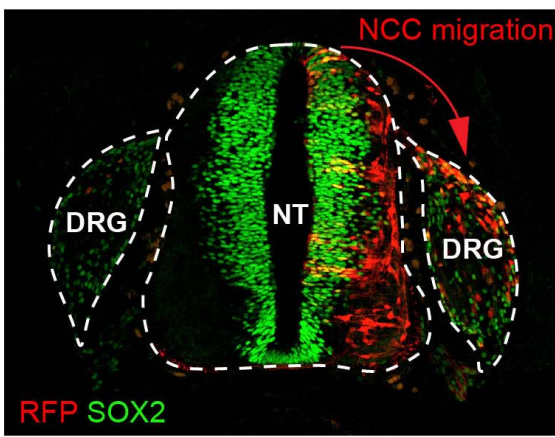

B
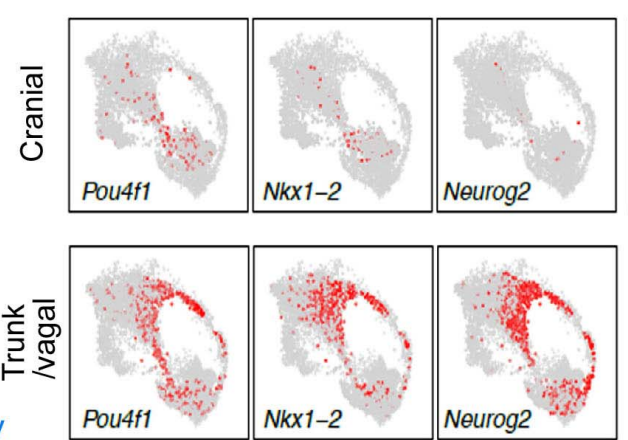

Mesenchymal regulators
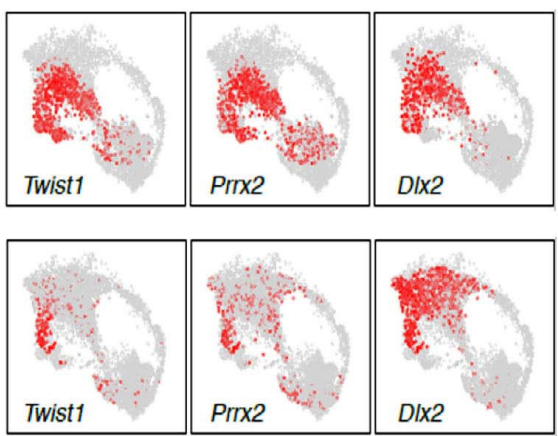

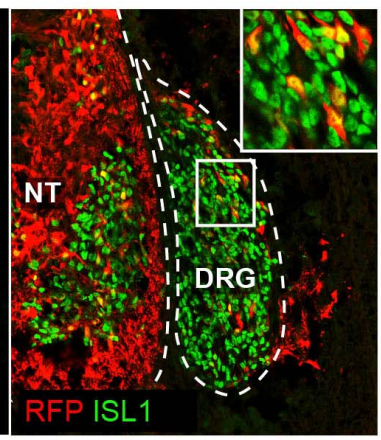

Electroporation of Twist1 + RFP plasmid into trunk NCC
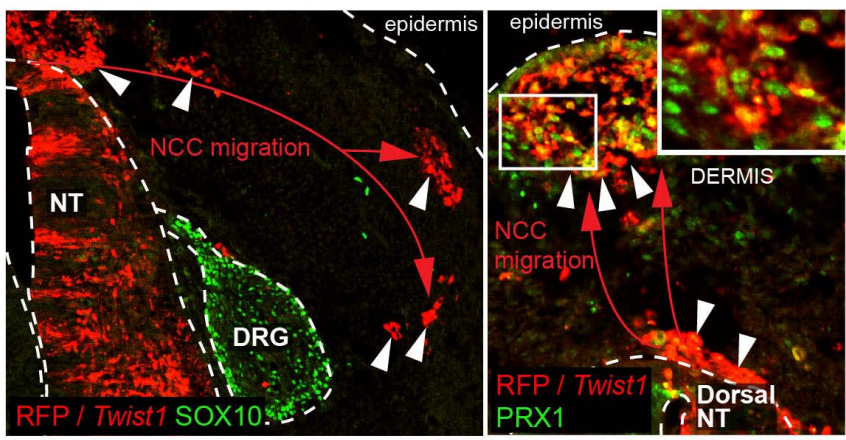

Fig. 6. Activation of early mesenchymal and sensory transcriptional programs. (A) Scatter plot contrasts expression changes accompanying NC transition from pre-EMT to migrating stages in cranial and trunk NC shows overall correlation ( $r=0.5)$. However, more up-regulated genes upon transition in trunk or in cranial compartments end up preferentially expressed in sensory and mesenchymal-autonomic fates, respectively.

(B) Mesenchymal regulators are activated early in cranial cells (top, only cranial expression shown), whereas sensory regulators are activated early in trunk cells (bottom, only trunk expression shown). (C) Immunofluorescence for SOX2, ISL1, and RFP (red fluorescent protein) after electroporation of control RFP-containing plasmid (left) versus after Twist1 electroporation (right) in the developing chicken embryo trunk shows ectopic mesenchymal induction upon Twist1 overexpression. NC, neural crest; NT, neural tube; NCC, neural crest cell; DRG, dorsal root ganglion.

\section{Discussion}

We used single-cell profiling to characterize spatiotemporal transcriptional dynamics of neural crest, showing progressive restrictions of cell fates, each realized through sequential phases of coactivation, biasing, and resolution of competing fates (Fig. 7A). Computational reconstruction of such differentiation trajectories from transcriptional similarity merely predicts likely lineage relationships, requiring additional validation (55). Here we relied on targeted lineage tracings of Neurog2, Phox $2 b$, and Prrxy to validate gradual restriction of the developmental potential predicted by the derived transcriptional tree (Fig. 7B). However, clonal tracing techniques that are being developed to progressively record clonal histories of individual cells (56) would be ideally suited for validation of a complex differentiation process such as the one that takes place in neural crest.

The process of delamination largely erases the transcriptional signatures that distinguish different anteroposterior neural crest levels within the neural tube and activates fate-specifying gene programs. Such dissipation of pre-EMT signatures is consistent with the transient phenotype ob- served by Simoes-Costa et al., who identified a cranial-specific pre-EMT gene regulatory network capable of transient activation of the mesenchymal properties in trunk, but unable to overcome the environmental signals and generate trunk mesenchyme (57). It indicates that acquisition of a fate likely requires appropriate cell-intrinsic state and extrinsic signaling during delamination. Consistently, we find that mesenchymal transcriptional bias emerges during delamination, indicating that this might be the point where mesenchymal potential is imbued to the neural crest. In support of this hypothesis, we noted that sustained overexpression of Trwist1, normally activated upon delamination only in the cranial compartment, is sufficient to reverse trunk crest developmental program to a mesenchymal route. This parallels previous observations in chordates, where misexpression of a tunicate Twist resulted in conversion of a cephalic melanocyte lineage into migrating ectomesenchyme (58). Twist1 might therefore be the ancient and sufficient factor defining the mesenchymal potential of migrating neural crest-a key step in the evolutionary development of the head in vertebrates.
The spatiotemporal data presented here may provide a resource for studies of neural crest biology and regulation, as well as investigations of neural crest-derived cancers and neurocristopathy-associated diseases. Although cell fate decisions in other systems may be based on different organizational principles, the molecular logic of cell fate decision characterized here for murine neural crest may also be shared by other organisms or tissues. Additional data, arising from analogous single-cell transcriptional studies or the new generalized lineage-tracing techniques, might soon allow such interorganismal comparisons to be performed.

\section{Materials and methods summary}

Neural crest cells were traced using the $W n t 1^{\text {TOMATO }}$ and $S O x 10^{\text {CreERT2 }}$;R26R ${ }^{\text {TOMATO }}$ strains from E8.5 to E10.5 mouse embryos. Embryos were collected and the $\mathrm{TOMATO}^{+}$parts of the tissue were selected after stereoscopical observation using ultraviolet illumination. The tissue was dissociated and $\mathrm{TOMATO}^{+}$cells were sorted by fluorescenceactivated cell sorting on a 384-well plate in singlecell mode. The sorted cells were lysed and their 
Fig. 7. Schematic representation of neural crest fate decisions. (A) Compared to a classical model (left), the model of NC development proposed here resolves a sequence of stages around delamination, heterogeneity of migratory progenitors, and sequential structure of lineage restrictions. (B) Upper: Individual binary decisions are resolved via a three-phase mechanism involving coactivation, biasing, and commitment. Lower: Lineage tracing shows that early activation of Neurog2 is linked to repression of melanocytes in multipotent progenitors, whereas Prrx1 and Phox $2 b$, involved in coactivation and biasing phases, mark downstream mesenchymal and autonomic fates with sporadic coexpression in bipotent population around fate bifurcation. (C) Distinct gene modules, activated upon delamination along the cranial to post-otic axis of the embryo, establish bias toward either mesenchymal (e.g., Twist1) or sensory neuronal fate.

\section{A Classical model of NC transitions}

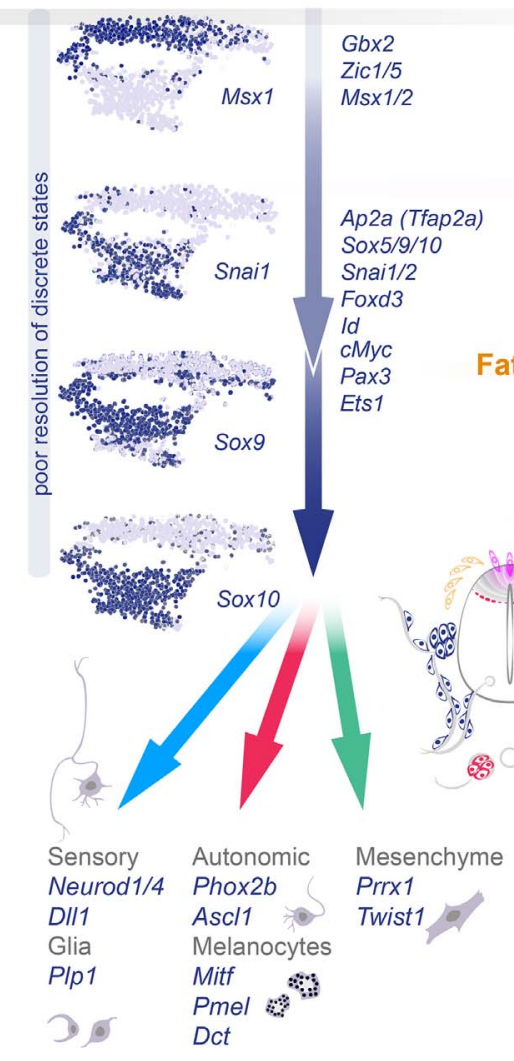

B
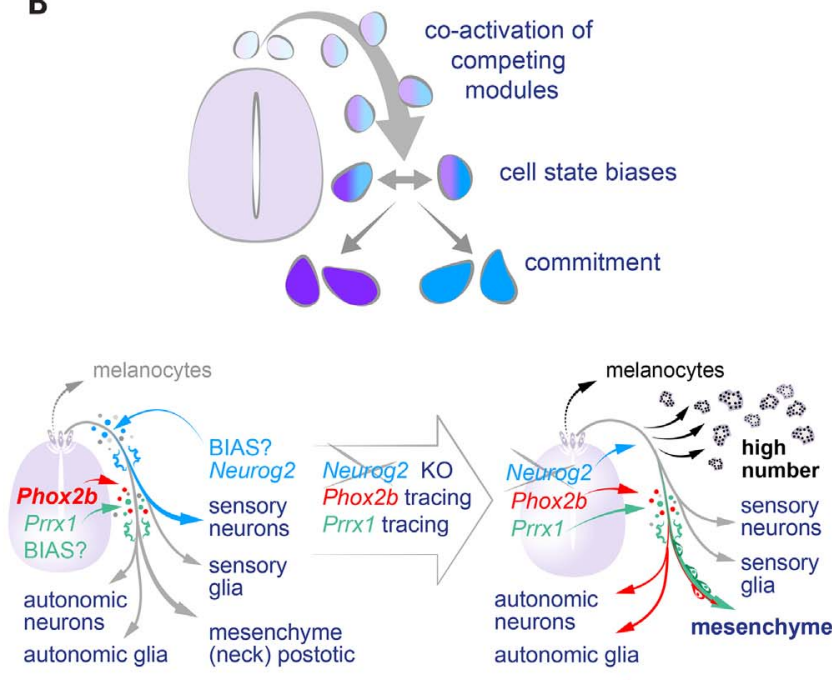

$$
\begin{aligned}
& \text { Prrxi } 1^{+} \text {cells commit } \\
& \text { to mesenchymal fate } \\
& \text { Phox } 2 b^{+} \text {cells largely } \\
& \text { commit to autonomic fate }
\end{aligned}
$$

\section{New model of NC development}

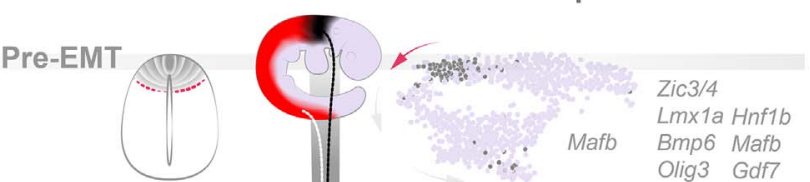

$\begin{array}{ll}\text { Bmp6 } & \text { Mafb } \\ \text { Olig3 } & \text { Gdf7 }\end{array}$

Delaminating/EMT
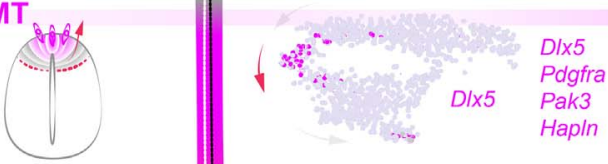

Pdgfra

Hapln

Ret

Ngfr

Mcam

$D \mid \times 2$
Melanocytes Mitf

Dct

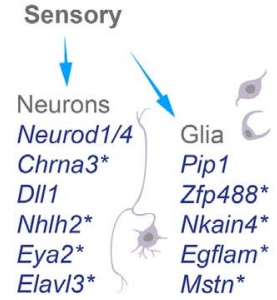

*-specific markers from unbiased clustering transcriptomes sequenced using the Smart-seq2 protocol. Individual single-cell datasets were preprocessed using PAGODA1 and PAGODA2 packages, which includes normalization, clustering of cells, and t-distributed stochastic neighbor embedding (t-SNE) for visualization (7). Joint analysis of multiple datasets was performed using the CONOS package (59).

Individual populations of clustered cells were validated using a variety of approaches. Major

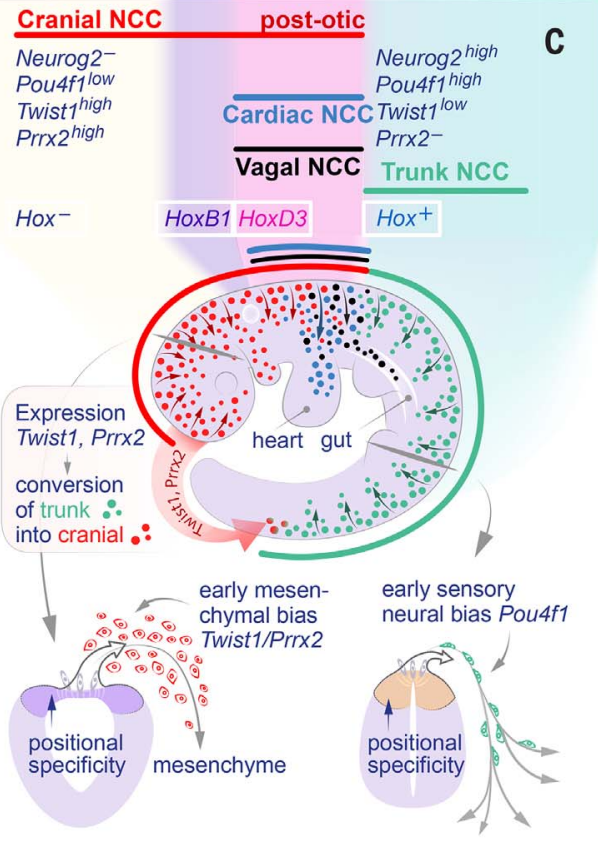

subpopulations (pre-EMT, delaminating and migrating neural crest, sensory neurons, autonomic neurons, and mesenchyme) were validated on E9.5 embryos using immunofluorescence and comparison with public in situ databases 
(Allen Brain Atlas and Eurexpress) $(17,18)$. Anatomical localization of the subpopulations was established using multiplexed in situ sequencing of 32 genes (19), followed by computational alignment of the single-cell transcriptional profiles with the spatially resolved in situ measurements. Expression patterns of specific markers of the sensory lineages were validated on E9.5 and E11.5 stages using RNAscope.

RNA velocity was used to assess transcriptional dynamics of cells (8). To quantify segregation of lineages, we adapted a computational method to reconstruct transcriptional trajectory tree from single-cell profiles using principal graph approach (20). Expression of transcription factors and their potential targets, predicted on the basis of DNA binding motifs, was analyzed to assess regulatory activity along the tree. We developed a computational method to analyze patterns of cell fate decisions, which tracks formation of fate-specific gene modules prior to fate bifurcations. Dynamics around major branches of neural crest fate acquisition (mesenchyme, sensory and autonomic neurogenesis) were further analyzed using lineage tracing with the Prrx1-Cre;R26R ${ }^{\text {mTMG }}$, Phox $2 b-C r e ; R 26 R^{\text {TOMATO }}$ and $A s c l 1^{\text {CreERT2 }}$;R26R ${ }^{\text {TOMATO }}$ mouse strains.

Additional validation included the analysis of knockin Neurog $2^{\text {CreERT2 }}$;R26R ${ }^{Y F P}$ mice (where Neurog2 is replaced by CreERT2, constituting homozygous embryos Neurog2 knockouts). Overexpression of Twist1, as well as CRISPR-Cas9mediated knockdown, performed as described in (60), in developing chicken embryos using in ovo electroporation, was used to validate Twist1 involvement in mesenchymal fate acquisition. Similarly, in ovo chicken electroporation was used to overexpress Prrx 2 (mesenchymal driver) and Crabp1 (driver of neuron-glia fate acquisition) and a dominant-negative version of Brn3c (avian homolog of Pou4fi).

\section{REFERENCES AND NOTES}

1. I. Imayoshi et al., Oscillatory control of factors determining multipotency and fate in mouse neural progenitors. Science 342, 1203-1208 (2013). doi: 10.1126/science.1242366; pmid: 24179156

2. C. Pina et al., Inferring rules of lineage commitment in haematopoiesis. Nat. Cell Biol. 14, 287-294 (2012). doi: 10.1038/ncb2442; pmid: 22344032

3. V. W. C. Yu et al., Epigenetic Memory Underlies Cell-Autonomous Heterogeneous Behavior of Hematopoietic Stem Cells. Cell 168, 944-945 (2017). doi: 10.1016/j.cell.2017.02.010; pmid: 28235203

4. D. R. Cordero et al., Cranial neural crest cells on the move: Their roles in craniofacial development. Am. J. Med. Genet. A. 155, 270-279 (2011). doi: 10.1002/ajmg.a.33702; pmid: 21271641

5. N. M. Le Douarin, The Neural Crest (Cambridge Univ. Press, 1982).

6. M. Simões-Costa, M. E. Bronner, Establishing neural crest identity: A gene regulatory recipe. Development 142, 242-257 (2015). doi: 10.1242/dev.105445; pmid: 25564621

7. J. Fan et al., Characterizing transcriptional heterogeneity through pathway and gene set overdispersion analysis. Nat. Methods 13, 241-244 (2016). doi: 10.1038/nmeth.3734; pmid: 26780092

8. G. La Manno et al., RNA velocity of single cells. Nature $\mathbf{5 6 0}$ 494-498 (2018). doi: 10.1038/s41586-018-0414-6; pmid: 30089906

9. M. E. Bronner, M. Simões-Costa, The Neural Crest Migrating into the Twenty-First Century. Curr. Top. Dev. Biol. 116, 115-134 (2016). doi: 10.1016/bs.ctdb.2015.12.003; pmid: 26970616
10. K. Nakata, T. Nagai, J. Aruga, K. Mikoshiba, Xenopus Zic3, a primary regulator both in neural and neural crest development. Proc. Natl. Acad. Sci. U.S.A. 94, 11980-11985 (1997). doi: 10.1073/pnas.94.22.11980; pmid: 9342348

11. K. F. Liem Jr., G. Tremml, T. M. Jessell, A role for the roof plate and its resident TGFbeta-related proteins in neuronal patterning in the dorsal spinal cord. Cell 91, 127-138 (1997). doi: 10.1016/S0092-8674(01)80015-5; pmid: 9335341

12. L. Lo, E. L. Dormand, D. J. Anderson, Late-emigrating neural crest cells in the roof plate are restricted to a sensory fate by GDF7. Proc. Natl. Acad. Sci. U.S.A. 102, 7192-7197 (2005). doi: 10.1073/pnas.0502581102; pmid: 15883363

13. K. J. Millen, J. H. Millonig, M. E. Hatten, Roof plate and dorsal spinal cord dl1 interneuron development in the dreher mutant mouse. Dev. Biol. 270, 382-392 (2004). doi: 10.1016/ j.ydbio.2004.03.008; pmid: 15183721

14. W. Yanfeng, J. P. Saint-Jeannet, P. S. Klein, Wnt-frizzled signaling in the induction and differentiation of the neural crest. BioEssays 25, 317-325 (2003). doi: 10.1002/bies.10255; pmid: 12655639

15. S. H. Kil, C. E. Krull, G. Cann, D. Clegg, M. Bronner-Fraser, The alpha4 subunit of integrin is important for neural crest cell migration. Dev. Biol. 202, 29-42 (1998). doi: 10.1006/ dbio.1998.8985; pmid: 9758701

16. S. Nakagawa, M. Takeichi, Neural crest emigration from the neural tube depends on regulated cadherin expression. Development 125, 2963-2971 (1998). pmid: 9655818

17. G. Diez-Roux et al., A high-resolution anatomical atlas of the transcriptome in the mouse embryo. PLOS Biol. 9 e1000582 (2011). doi: 10.1371/journal.pbio.1000582; pmid: 21267068

18. E. S. Lein et al., Genome-wide atlas of gene expression in the adult mouse brain. Nature 445, 168-176 (2007). doi: 10.1038/ nature05453; pmid: 17151600

19. R. Ke et al., In situ sequencing for RNA analysis in preserved tissue and cells. Nat. Methods 10, 857-860 (2013). doi: 10.1038/nmeth.2563; pmid: 23852452

20. Q. Mao, L. Wang, I. W. Tsang, Y. Sun, Principal Graph and Structure Learning Based on Reversed Graph Embedding. IEEE Trans. Pattern Anal. Mach. Intell. 39, 2227-2241 (2017) doi: 10.1109/TPAMI.2016.2635657; pmid: 28114001

21. A. Baggiolini et al., Premigratory and migratory neural crest cells are multipotent in vivo. Cell Stem Cell 16, 314-322 (2015) doi: 10.1016/i.stem.2015.02.017; pmid: 25748934

22. M. Bronner-Fraser, S. Fraser, Developmental potential of avian trunk neural crest cells in situ. Neuron 3, 755-766 (1989). doi: 10.1016/0896-6273(89)90244-4; pmid: 2484346

23. N. M. Le Douarin, G. W. Calloni, E. Dupin, The stem cells of the neural crest. Cell Cycle 7, 1013-1019 (2008). doi: 10.4161/ cc.7.8.5641; pmid: 18414040

24. L. Zurkirchen, L. Sommer, Quo vadis: Tracing the fate of neural crest cells. Curr. Opin. Neurobiol. 47, 16-23 (2017) doi: 10.1016/j.conb.2017.07.001; pmid: 28753439

25. A. E. Lewis, H. N. Vasudevan, A. K. O’Neill, P. Soriano, J. O. Bush, The widely used Wnt1-Cre transgene causes developmental phenotypes by ectopic activation of Wnt signaling. Dev. Biol. 379, 229-234 (2013). doi: 10.1016/j.ydbio.2013.04.026; pmid: 23648512

26. M. Minoux et al., Gene bivalency at Polycomb domains regulates cranial neural crest positional identity. Science 355, eaal2913 (2017). doi: 10.1126/science.aal2913; pmid: 28360266

27. H. Suzuki et al., The transcriptional network that controls growth arrest and differentiation in a human myeloid leukemia cell line. Nat. Genet. 41, 553-562 (2009). doi: 10.1038/ng.375; pmid: 19377474

28. R. A. Stewart et al., Zebrafish foxd3 is selectively required for neural crest specification, migration and survival. Dev. Biol. 292, 174-188 (2006). doi: 10.1016/j.ydbio.2005.12.035; pmid: 16499899

29. N. A. Mundell, P. A. Labosky, Neural crest stem cell multipotency requires Foxd3 to maintain neural potential and repress mesenchymal fates. Development 138, 641-652 (2011). doi: 10.1242/dev.054718; pmid: 21228004

30. J. Jacob et al., Insml (IA-1) is an essential component of the regulatory network that specifies monoaminergic neuronal phenotypes in the vertebrate hindbrain. Development 136, 2477-2485 (2009). doi: 10.1242/dev.034546; pmid: 19542360

31. O. Ma, C. Fode, F. Guillemot, D. J. Anderson, Neurogenin1 and neurogenin2 control two distinct waves of neurogenesis in developing dorsal root ganglia. Genes Dev. 13, 1717-1728 (1999). doi: 10.1101/gad.13.13.1717: pmid: 10398684
32. M. Zirlinger, L. Lo, J. McMahon, A. P. McMahon, D. J. Anderson, Transient expression of the bHLH factor neurogenin-2 marks a subpopulation of neural crest cells biased for a sensory but not a neuronal fate. Proc. Natl. Acad. Sci. U.S.A. 99, 8084-8089 (2002). doi: 10.1073/pnas.122231199; pmid: 12060754

33. M. Florio et al., Neurogenin 2 regulates progenitor cell-cycle progression and Purkinje cell dendritogenesis in cerebellar development. Development 139, 2308-2320 (2012). doi: 10.1242/dev.075861; pmid: 22669821

34. T. J. Stuhlmiller, M. I. García-Castro, Current perspectives of the signaling pathways directing neural crest induction. Cell. Mol. Life Sci. 69, 3715-3737 (2012). doi: 10.1007/ s00018-012-0991-8; pmid: 22547091

35. M. Crow, A. Paul, S. Ballouz, Z. J. Huang, J. Gillis, Exploiting single-cell expression to characterize co-expression replicability. Genome Biol. 17, 101 (2016). doi: 10.1186/ s13059-016-0964-6; pmid: 27165153

36. C. Trapnell, Defining cell types and states with single-cell genomics. Genome Res. 25, 1491-1498 (2015). doi: 10.1101/ gr.190595.115; pmid: 26430159

37. E. Abranches et al., Stochastic NANOG fluctuations allow mouse embryonic stem cells to explore pluripotency. Development 141, 2770-2779 (2014). doi: 10.1242/dev.108910; pmid: 25005472

38. M. Hu et al., Multilineage gene expression precedes commitment in the hemopoietic system. Genes Dev. 11 774-785 (1997). doi: 10.1101/gad.11.6.774; pmid: 9087431

39. S. Huang, Y. P. Guo, G. May, T. Enver, Bifurcation dynamics in lineage-commitment in bipotent progenitor cells. Dev. Biol. 305, 695-713 (2007). doi: 10.1016/j.ydbio.2007.02.036; pmid: 17412320

40. M. Thomson et al., Pluripotency factors in embryonic stem cells regulate differentiation into germ layers. Cell 145 875-889 (2011). doi: 10.1016/j.cell.2011.05.017; pmid: 21663792

41. J. A. Briggs et al., The dynamics of gene expression in vertebrate embryogenesis at single-cell resolution. Science 360 , eaar5780 (2018). doi: 10.1126/science.aar5780; pmid: 29700227

42. J. A. Farrell et al., Single-cell reconstruction of developmental trajectories during zebrafish embryogenesis. Science 360 eaar3131 (2018). doi: 10.1126/science.aar3131; pmid: 29700225

43. S. Semrau et al., Dynamics of lineage commitment revealed by single-cell transcriptomics of differentiating embryonic stem cells. Nat. Commun. 8, 1096 (2017). doi: 10.1038/ s41467-017-01076-4; pmid: 29061959

44. V. Boeva et al., Heterogeneity of neuroblastoma cell identity defined by transcriptional circuitries. Nat. Genet. 49 1408-1413 (2017). doi: 10.1038/ng.3921; pmid: 28740262

45. T. van Groningen et al., Neuroblastoma is composed of two super-enhancer-associated differentiation states. Nat. Genet. 49, 1261-1266 (2017). doi: 10.1038/ng.3899; pmid: 28650485

46. A. Pattyn, X. Morin, H. Cremer, C. Goridis, J. F. Brunet, Expression and interactions of the two closely related homeobox genes Phox2a and Phox2b during neurogenesis. Development 124, 4065-4075 (1997). pmid: 9374403

47. A. E. Doufexi, M. Mina, Signaling pathways regulating the expression of Prx1 and Prx2 in the chick mandibula mesenchyme. Dev. Dyn. 237, 3115-3127 (2008). doi: 10.1002/ dvdy.21762; pmid: 18942149

48. C. Gans, R. G. Northcutt, Neural crest and the origin of vertebrates: A new head. Science 220, 268-273 (1983) doi: 10.1126/science.220.4594.268; pmid: 17732898

49. I. Espinosa-Medina et al., Dual origin of enteric neurons in vagal Schwann cell precursors and the sympathetic neural crest. Proc. Natl. Acad. Sci. U.S.A. 114, 11980-11985 (2017). doi: 10.1073/pnas.1710308114; pmid: 29078343

50. M. Scheffer et al., Anticipating critical transitions. Science 338, 344-348 (2012). doi: 10.1126/science.1225244 pmid: 23087241

51. E. J. Hutchins et al., Migration and diversification of the vagal neural crest. Dev. Biol. 444 (suppl. 1), S98-S109 (2018). doi: 10.1016/j.ydbio.2018.07.004; pmid: 29981692

52. F. J. de Lange et al., Lineage and morphogenetic analysis of the cardiac valves. Circ. Res. 95, 645-654 (2004). doi: 10.1161/ 01.RES.0000141429.13560.cb; pmid: 15297379

53. H. Bildsoe et al., Requirement for Twist1 in frontonasal and skull vault development in the mouse embryo. Dev. Biol. 331, 176-188 (2009). doi: 10.1016/j.ydbio.2009.04.034 pmid: 19414008

54. J. W. Vincentz et al., Twist1 controls a cell-specification switch governing cell fate decisions within the cardiac neural crest. PLOS Genet. 9, e1003405 (2013). doi: 10.1371/journal. pgen.1003405; pmid: 23555309 
55. C. Weinreb, S. Wolock, B. K. Tusi, M. Socolovsky, A. M. Klein, Fundamental limits on dynamic inference from single-cell snapshots. Proc. Natl. Acad. Sci. U.S.A. 115, E2467-E2476 (2018). doi: 10.1073/pnas.1714723115; pmid: 29463712

56. L. Kester, A. van Oudenaarden, Single-Cell Transcriptomics Meets Lineage Tracing. Cell Stem Cell 23, 166-179 (2018). doi: 10.1016/j.stem.2018.04.014; pmid: 29754780

57. M. Simoes-Costa, M. E. Bronner, Reprogramming of avian neural crest axial identity and cell fate. Science 352 , 1570-1573 (2016). doi: 10.1126/science.aaf2729; pmid: 27339986

58. P. B. Abitua, E. Wagner, I. A. Navarrete, M. Levine, Identification of a rudimentary neural crest in a non-vertebrate chordate. Nature 492, 104-107 (2012). doi: 10.1038/nature11589; pmid: 23135395

59. N. Barkas et al., Wiring together large single-cell RNA-seq sample collections. bioRxiv (2018). doi: 10.1101/460246

60. S. Gandhi, M. L. Piacentino, F. M. Vieceli, M. E. Bronner, Optimization of CRISPR/Cas9 genome editing for loss-of-function in the early chick embryo. Dev. Biol. 432, 86-97 (2017). doi: 10.1016/j.ydbio.2017.08.036; pmid: 29150011

\section{ACKNOWLEDGMENTS}

We thank the Eukaryotic Single Cell Genomics Facility for the single-cell RNA-seq and the in situ sequencing pilot facility at the Science for Life Laboratory, Sweden (for technical assistance with the in situ sequencing experiments). We also thank 0 . Kharchenko for help with illustrations and V. Petukhov for assistance with computational analysis of in situ sequencing data. Funding: I.A. was funded by a Swedish Research Council (Vetenskapsradet, VR) grant, ERC Consolidator grant "STEMMING-FROM-NERVE" N647844, the Paradifference Foundation, and the Bertil Hallsten Research Foundation. P.V.K. was supported by NSF-14-532 CAREER award and NIH R01HL131768. M.N. was funded by a Swedish Research Council (Vetenskapsradet) grant 2016-03645, the Knut and Alice Wallenberg Foundation, and Familjen Erling Perssons stiftelse. V.D. was supported by a Russian Science Foundation grant (18-7510005, immunochemistry) and the Swedish Research Council (2015-03387). M.E.K. was supported by Stiftelsen Riksbankens Jubileumsfond (Erik Rönnbergs fond stipend). N.A. was supported by RSF grant 16-15-10237. Author contributions: M.K., M.E.K., J.P., L.E., N.A., Y.Y., M.H., V.D., M.F., M.L.P., F.B., C.Y., X.Q., W.-Y.H., and L.C. acquired all biological data and performed the relevant analysis. R.S., P.V.K., and T.C. performed computational analysis of single-cell data. R.S. and P.V.K. developed computational methods. R.S. and M.M.H. performed computational analysis of in situ sequencing data. C.B., M.N., M.E.B., D.A.G., J.-F.B., G.G.C., P.E., K.F., P.V.K., and I.A. gave feedback on experimental aspects, supervised experimental approaches, and implemented the data interpretation. R.S., M.K., M.E.K., and J.P. made all figures containing data and resulting analysis (except Fig. 7). R.S., M.K., M.E.K., P.V.K., and I.A. designed the study, organized experimental work, and wrote the manuscript. All authors provided feedback on figures, manuscript composition, and structure. Competing interests: M.N. holds shares in Cartana $A B$, a company commercializing in situ sequencing reagents. Other authors declare no conflicts of interest. Data and materials availability: All single-cell RNA-seq datasets have been deposited in the GEO under accession code GSE129114. Processed data, code, supplementary materials, and interactive views of datasets can be accessed on the authors' website: http://pklab.med.harvard.edu/ruslan/neural.crest.html. These URLs will be maintained exactly as they are for at least 5 years with no changes. The Sox10 $0^{\text {CreERT2 }}$ strain is available from the laboratory of Vassilis Pachnis (The Francis Crick Institute, UK) under a material transfer agreement with the institution. All other data needed to evaluate the conclusions in the paper are present in the paper or the supplementary materials.

\section{SUPPLEMENTARY MATERIALS}

science.sciencemag.org/content/364/6444/eaas9536/suppl/DC1 Materials and Methods

Supplementary Text

Figs. S1 to S12

Data S1 to S12

Data Tables S1 to S11

References (61-75)

9 January 2018; resubmitted 12 December 2018

Accepted 10 April 2019

10.1126/science.aas9536 


\section{Science}

\section{Spatiotemporal structure of cell fate decisions in murine neural crest}

Ruslan Soldatov, Marketa Kaucka, Maria Eleni Kastriti, Julian Petersen, Tatiana Chontorotzea, Lukas Englmaier, Natalia Akkuratova, Yunshi Yang, Martin Häring, Viacheslav Dyachuk, Christoph Bock, Matthias Farlik, Michael L. Piacentino, Franck Boismoreau, Markus M. Hilscher, Chika Yokota, Xiaoyan Qian, Mats Nilsson, Marianne E. Bronner, Laura Croci, Wen-Yu Hsiao, Jean-Francois Brunet, Gian Giacomo Consalez, Patrik Ernfors, Kaj Fried, Peter V. Kharchenko and Igor Adameyko

Science 364 (6444), eaas9536.

DOI: $10.1126 /$ science.aas9536

\section{Binary decisions refine fate decisions}

Neural crest cells develop into tissues ranging from craniofacial bones to peripheral neurons. Combining single-cell RNA sequencing with spatial transcriptomics, Soldatov et al. analyzed how neural crest cells in mouse embryos decide among the various fates available to them (see the Perspective by Mayor). These multipotent cells become biased toward a given fate early on and step through a progression of binary decisions as their fate is refined. Competing fate programs coexist until increased synchronization favors one and repression disfavors the other.

Science, this issue p. eaas9536; see also p. 937

ARTICLE TOOLS

SUPPLEMENTARY

MATERIALS

RELATED

CONTENT

REFERENCES

PERMISSIONS http://science.sciencemag.org/content/364/6444/eaas9536

http://science.sciencemag.org/content/suppl/2019/06/05/364.6444.eaas9536.DC1

http://science.sciencemag.org/content/sci/364/6444/937.full

This article cites 74 articles, 30 of which you can access for free http://science.sciencemag.org/content/364/6444/eaas9536\#BIBL

http://www.sciencemag.org/help/reprints-and-permissions

Use of this article is subject to the Terms of Service

Science (print ISSN 0036-8075; online ISSN 1095-9203) is published by the American Association for the Advancement of Science, 1200 New York Avenue NW, Washington, DC 20005. 2017 (C) The Authors, some rights reserved; exclusive licensee American Association for the Advancement of Science. No claim to original U.S. Government Works. The title Science is a registered trademark of AAAS. 